\title{
The transcriptomic response to a short day to long day shift in leaves of the reference legume Medicago truncatula
}

\author{
Geoffrey Thomson ${ }^{\text {Corresp.. }}{ }^{1}$, James Taylor ${ }^{1}$, Joanna Putterill ${ }^{\text {Corresp. } 1}$ \\ ${ }^{1}$ School of Biological Sciences, University of Auckland, Auckland, New Zealand \\ Corresponding Authors: Geoffrey Thomson, Joanna Putterill \\ Email address: gtho123@aucklanduni.ac.nz, j.putterill@auckland.ac.nz
}

Photoperiodic flowering aligns plant reproduction to favourable seasons of the year to maximise successful production of seeds and grains. However understanding of this process in the temperate legumes of the Fabaceae family, which are important both agriculturally and ecologically, is incomplete. Previous work in the reference legume Medicago truncatula has shown that the FT-like gene MtFTa1 is a potent floral activator. While MtFTa1 is upregulated by long-day photoperiods (LD) and vernalisation, the molecular basis of this is unknown as functional homologues of key regulatory genes present in other species, notably CONSTANS in A. thaliana, have not been identified. In LD MtFTa1 maintains a near constant diurnal pattern of expression unlike its homologue $F T$ in A. thaliana, which has a notable peak in expression at dusk. This suggests a different manner of regulation. Furthermore, M. truncatula possesses other FT-like genes such as two LD induced MtFTb genes which may also act in the regulation of flowering time. MtFTb genes have a diurnal pattern of expression with peaks at both four and sixteen hours after dawn. This study utilises RNA-Seq to analyse the transcriptome of M. truncatula leaves to identify genes which may regulate or be co-expressed with these FT-like genes following a shift from short-day photoperiods to inductive long-days. Specifically this study focuses on the first four hours of the day in the young leaves, which coincides with the first diurnal peak of the FTb genes. Following differential expression analysis at each timepoint, genes which alter their pattern of expression are distinguished from those which just alter their magnitude of expression (and those that do neither). It goes on to categorise these genes into groups with similar patterns of expression using c-means clustering and identifies a number of potential candidate photoperiod flowering time genes for future studies to consider. 


\title{
The transcriptomic response to a short day to long day shift in leaves of the reference legume Medicago truncatula
}

\author{
Geoffrey Thomson ${ }^{1}$, James Taylor ${ }^{1}$, and Joanna Putterill ${ }^{1}$ \\ 'School of Biological Sciences, The University of Auckland, Auckland, New Zealand \\ 6 Corresponding author: \\ 7 Geoffrey Thomson, Joanna Putterill \\ 8 Email address: gtho123@aucklanduni.ac.nz, j.putterill@auckland.ac.nz
}

\section{ABSTRACT}

\begin{abstract}
Photoperiodic flowering aligns plant reproduction to favourable seasons of the year to maximise successful production of seeds and grains. However understanding of this process in the temperate legumes of the Fabaceae family, which are important both agriculturally and ecologically, is incomplete. Previous work in the reference legume Medicago truncatula has shown that the FT-like gene MtFTa1 is a potent floral activator. While MtFTa1 is upregulated by long-day photoperiods (LD) and vernalisation, the molecular basis of this is unknown as functional homologues of key regulatory genes present in other species, notably CONSTANS in A. thaliana, have not been identified. In LD MtFTa1 maintains a near constant diurnal pattern of expression unlike its homologue $F T$ in $A$. thaliana, which has a notable peak in expression at dusk. This suggests a different manner of regulation. Furthermore, $M$. truncatula possesses other FT-like genes such as two LD induced MtFTb genes which may also act in the regulation of flowering time. MtFTb genes have a diurnal pattern of expression with peaks at both four and sixteen hours after dawn. This study utilises RNA-Seq to analyse the transcriptome of $M$. truncatula leaves to identify genes which may regulate or be co-expressed with these FT-like genes following a shift from short-day photoperiods to inductive long-days. Specifically this study focuses on the first four hours of the day in the young leaves, which coincides with the first diurnal peak of the FTb genes. Following differential expression analysis at each timepoint, genes which alter their pattern of expression are distinguished from those which just alter their magnitude of expression (and those that do neither). It goes on to categorise these genes into groups with similar patterns of expression using c-means clustering and identifies a number of potential candidate photoperiod flowering time genes for future studies to consider.
\end{abstract}

\section{INTRODUCTION}

The regulation of flowering controls the important developmental shift between the vegetative and reproductive growth phases of the plant, aligning plant sexual reproduction with favourable seasonal environmental variation. This facilitates successful pollination and the maximizing of crop productivity and yield. In many temperate climate species, such as the winter annual varieties of the well-studied Brassicaceae species Arabidopsis thaliana (L.) Heynh., the primary determinants of flowering time are long-day (LD) photoperiod (daylength) conditions and vernalisation (prolonged exposure to cold temperatures) for which the molecular pathways underlying these responses are well understood (Andrés and Coupland, 2012).

In A. thaliana, LD conditions are perceived in the leaves and culminates in the activation of the floral integrator gene FLOWERING LOCUS T (FT) (Turck et al., 2008). Specifically, FT is activated by circadian and light signals aligning which facilitates the formation of the GIGANTEA-FLAVINBINDING KELCH REPEAT, F-BOX 1 (GI-FKF1) complex. This complex degrades CYCLING DOF FACTOR (CDF) transcription factors which otherwise form a complex with a TOPLESS (TPL) protein to repress the expression of the transcription factor CONSTANS $(C O)$. This gene encodes a B-box class protein with a CCT domain (Putterill et al., 1995; Goralogia et al., 2017). The stabilisation of CO protein in the late afternoon of LD results in it acting as a subunit of a NUCLEAR FACTOR-Y (NF-Y) pioneer 
transcription factor complex which directly activates FT (Andrés and Coupland, 2012; Gnesutta et al., 2017). CDF proteins are also able to repress $F T$ directly (Song et al., 2015). FT protein is the principal mobile floral signal (florigen) which is transported to the shoot apical meristem via the phloem to induce flowering via activation of a second floral integrator gene SUPPRESSOR OF OVEREXPRESSION OF CONSTANS 1 (SOCl) and the floral meristem identity gene APETALAl. This results in a state of floral commitment and the development of flowers (Turck et al., 2008).

Beyond A. thaliana, the presence of $F T$ orthologues integrating environmental signals and regulating flowering appears to be widely conserved (Ballerini and Kramer, 2011; Wickland and Hanzawa, 2015; Putterill and Varkonyi-Gasic, 2016). However the conservation of other elements of the pathway is not as strong. Recent work by Simon et al. (2015) suggest that the central role $C O$ plays in the photoperiod pathway in A. thaliana evolved within the Brassicaceae following a gene duplication. Thus CO-like (COL) genes regulating $F T$ homologues is not universal and their activity in some other species (e.g. Oryza sativa L.; rice; Hayama et al., 2003) are the result of convergent evolution. Examples of species in which COL genes do not appear to regulate flowering time include Japanese morning glory (Ipomoea nil (L.) Roth) and temperate Fabaceae family (legume) species such as pea (Pisum sativum L.) and Medicago truncatula Gaertn. Notably, a recent survey of $C O L$ genes in M. truncatula found no evidence of them acting as a photoperiodic switch (Wong et al., 2014) and the gene expression of the closest $C O L$ homologue in pea, was not altered in photoperiodic flowering time mutants. These include the recessive late bloomer 1 (late 1) mutant which disrupts the orthologue of GI (Hecht et al., 2007; Liew et al., 2009) and a dominant late flowering mutant late 2 which has been mapped to a $C D F$ gene $P s C D F c$ (Ridge et al., 2016). This $P s c d f c$ mutant encodes a protein which has lost the ability to interact with the FKF1 and exhibits reduced expression of FT-like genes (Ridge et al., 2016). Whether the regulation of the PsFT-like genes by PsCDFc is direct or not remains unknown.

Nevertheless, in many species the regulation of $F T$ orthologues exhibit significant commonalities such as genes containing CCT and/or B-box domains integrating the photoperiod and vernalisation signals. For instance, in the Pooid grasses such as wheat (Triticum spp.) prior to vernalisation the repression of the FT-like gene is maintained by a pair of ZCCT proteins, which contain both CCT and B-box domains, in complex with NF-Y subunits (Song et al., 2015; Li et al., 2011). Then in inductive LD conditions upregulation of the FT orthologue requires the PHOTOPERIOD 1 (PPDI) gene, which encodes a CCT domain (Shaw et al., 2013; Pearce et al., 2017). In addition genes encoding CCT and B-Box domains are important in the cultivated varieties of the LD responsive sugar beet (Beta vulgaris L.) (Pin et al., 2012; Dally et al., 2014).

The lack of a direct upstream regulator of FT-like genes in temperate legumes means that, despite good progress, the understanding of how flowering time in this family is incomplete (Putterill et al., 2013; Weller and Ortega, 2015). Legumes are an ecologically diverse plant family (The Legume Phylogeny Working Group, 2013) and include a number of dietary staple crops. In an agricultural context these crops reduce the need for fertilizer use via nitrogen fixation (Vance, 2001). Like A. thaliana, many temperate legume species, including $M$. truncatula, accelerate their flowering in response to vernalisation and LD conditions (Highkin, 1956; Summerfield et al., 1985; Roberts et al., 1985; Laurie et al., 2011; Weller and Ortega, 2015; Ridge et al., 2017). Classically pea has been most intensively studied to analyse photoperiodic flowering in temperate legumes but has recently been complemented by the study of other species, such as M. truncatula for which considerable genetic and genomic resources exist (Tadege et al., 2008; Young et al., 2011; Tang et al., 2014).

Analysis of flowering time mutants in pea has demonstrated that some members of the photoperiod pathway, such as the photoreceptors and components of the circadian clock, are largely conserved with $A$. thaliana (Weller and Ortega, 2015). In addition, FT-like homologues have been characterised in several legume species with most having multiple copies which fall into three sub-clades (Laurie et al., 2011; Hecht et al., 2011). In pea and M. truncatula the FT-like gene FTal is a potent floral inducer whose expression is elevated in LD (Laurie et al., 2011; Hecht et al., 2011). However unlike $F T$ in A. thaliana, MtFTal (Medtr7g084970) does not possess a diurnal pattern and instead exhibits a near constant level of expression once induced in LD (Laurie et al., 2011) suggesting that the mechanisms of regulation between $F T$ and $F T a l$ likely differ significantly. Moreover grafting experiments in pea between flowering time mutants suggest that additional floral stimuli exist (Hecht et al., 2011).

Good candidates for secondary floral stimuli are the FTb genes that, like FTal, are upregulated in LD and capable of complementing an A. thaliana ft-1 mutant (just MtFTb1 in M. truncatula, Laurie et al., 
2011; Hecht et al., 2011). Distinctively, MtFTb genes have a diurnal pattern of expression in LD and peak twice, at ZT4 and ZT16 (ZT is zeitgeber time where ZT0 is subjective dawn at lights on). This pattern of expression is similar to that of $F T$ in A. thaliana under "natural" LD conditions (Song et al., 2018). Another legume FT-like gene which may play a role in flowering time is FTa2 which in M. truncatula it is mostly expressed in short-day (SD) photoperiod conditions consistent with a floral repressor (Laurie et al., 2011). However whether any FT-like genes other than FTal regulate flowering time in either pea or $M$. truncatula remains to be demonstrated.

Downstream of FT-like genes the regulation of flowering in temperate legumes is similar overall to that of A. thaliana, albeit complicated by several genes being present in multiple copies with potential functional redundancies. For example, three MtSOCl-like genes depend on MtFTal for the extent and timing of their expression, although Mtsocla mutants do show delayed flowering (Fudge et al., 2018; Jaudal et al., 2018). The genes involved in inflorescence development are similar to that of A. thaliana (Cheng et al., 2018).

Overall, while some components of the A. thaliana photoperiodic flowering model appear to be conserved in temperate legumes, other aspects differ. Specifically, what factors act immediately upstream of FT-like genes in the photoperiodic flowering of temperate legumes remain unknown. In light of the gap in understanding, we take a transcriptomic approach to identify additional candidate regulators of photoperiodic flowering in M. truncatula. In two experiments we target genes expressed in a similar, or opposite, manner to LD induced FT-like genes with the aim of identifying candidate regulators. Plants were shifted from SD ( 8 hours light/16 hour dark) to LD (16 hour light/8 hour dark) conditions and gene expression changes analysed in the first four hours of the diurnal cycle; at dawn (ZT0), two hours after dawn (ZT2) and four hours after dawn (ZT4) during which time the LD induced FT-like genes are expressed. These timepoints capture the constant induction of MtFTal in LD and target the first diurnal peak of MtFTb1 and MtFTb2 at ZT4 also in LD (Laurie et al., 2011).

\section{MATERIALS AND METHODS}

\section{Growth of plants and tissue sampling}

M. truncatula cv 'Jester' (Hill, 2000) seeds were scarified by softly scraping them between two pieces of sand paper (grade P160). The seeds were then germinated at $15^{\circ} \mathrm{C}$ in gently shaking tubes of water and dark conditions for 24 hours. Germinated seeds were then vernalised by being transferred to damp petri dishes and incubated at $4{ }^{\circ} \mathrm{C}$ for a further 25 days. The seedlings were subsequently planted in seed raising mix (Daltons Ltd., NZ) in individual cell pots and grown in growth cabinets at $22^{\circ} \mathrm{C}$ under 240 $\mu \mathrm{Mm}^{-2} \mathrm{sec}^{-1}$ cool white fluorescent light. This was in accordance with Institutional Biological Safety Committee approval GMO08-UA006. Soil was kept moist with a complete liquid nutrient media (without $\mathrm{Na}_{2} \mathrm{SiO}_{3}$; Gibeaut et al., 1997). In the two experiments plants were grown in SD conditions (8 hours light/16 hour dark) until they were 10 days old and were then shifted at ZT8 into LD conditions (16 hours light/8 hour dark) for 3 days in experiment 1 (harvest at ZT0 and ZT2) or 5 days in experiment 2 (harvests at ZT4). Three biological replicates were taken each consisting of two pooled trifoliate leaves from different non-adjacent plants giving a total of 18 samples. Only the first trifoliate leaf to unfurl was sampled from a given plant. Samples were immediately frozen in liquid nitrogen.

\section{RNA extraction and sequencing}

The 18 frozen leaf samples were ground using five $3 \mathrm{~mm}$ sterilised ball bearings per sample and a Geno/Grinder ${ }^{\circledR} 2010$ (SPEX ${ }^{\circledR}$ SamplePrep, USA). Total RNA was then extracted from the ground samples using the RNeasy Plant Mini Kit (Qiagen, Germany) following the manufacturer's instructions and the quantities and qualities of the extracted RNA were then measured using a Bioanalyzer 2100 (Agilent Technologies, USA). Samples were then sent to the Otago Genomics Facility (www • otago. ac.nz/genomics/index.html) and RNA-Seq libraries were prepared. The first experiment (ZT0 and ZT2 samples) used the ScriptSeq Complete Kit (Plant) (100 bp reads; Illumina Inc., USA) while the second experiment (ZT4 samples) used TruSeq Stranded mRNA libraries (120 bp reads; Illumina Inc., USA). Each experiment was sequenced on a single lane of a HiSeq2000 (Illumina Inc., USA).

\section{Complementary DNA synthesis and RT-qPCR analysis}

Following RNA extraction, $8 \mu \mathrm{g}$ of RNA was treated with the TURBO DNA-free ${ }^{\mathrm{TM}} \mathrm{Kit}_{\text {(Invitrogen }}{ }^{\mathrm{TM}}$, USA) . First-strand complementary DNA (cDNA) was then synthesised using $1 \mu \mathrm{g}$ of DNase treated 
RNA using SuperScript ${ }^{\mathrm{TM}}$ IV Reverse Transcriptase (Invitrogen ${ }^{\mathrm{TM}}$, USA) using an oligo dT primer following the manufacturers instructions. At this point a control reaction where the reverse transcriptase is omitted was run, one reaction per set of replicate samples. When tested alongside synthesised cDNA these reactions control for the presence of genomic DNA contamination.

Measurement of relative abundances of cDNA, as a measure of gene expression, was done using Real time quantitative PCR (RT-qPCR). In this assay $10 \mu 1$ reactions using Power SYBR ${ }^{\mathrm{TM}}$ Green PCR Master Mix and $2 \mu \mathrm{l}$ of diluted cDNA (cDNA was diluted 20x prior to RT-qPCR). Control reactions using water, as well as the cDNA reactions which lacked reverse transcriptase, were run simultaneously. RT-qPCR experiments were assembled on 384-well plates and run on an Applied Biosystems 7900HT Sequence Detection System.

Analysis of the RT-qPCR data was done using the $2^{-\Delta \Delta C t}$ algorithm (Livak and Schmittgen, 2001). This utilised either Medtr7g089120 a TUBULIN BETA-1 CHAIN gene or Medtr6g084690 a SERINE/THREONINE PROTEIN PHOSPHATASE 2A REGULATORY SUBUNIT (PP2A; previously known as PROTODERMAL FACTOR 2) as reference genes (Kakar et al., 2008). Primers used are available in Table S2.

\section{RNA-Seq analysis}

Read trimming of the the FASTQ files was then performed using BBDuk tool in the BBTools suite (v37.54; Bushnell, 2018). This removed the sequencing adapters and low quality sequence (Phred $=20$ ) and retained only reads which were at least 36 bases in length. Furthermore read pairs lacking one of the pair were discarded. Transcripts were then quantified using Salmon (v0.8.2; Patro et al., 2017) which uses quasi-mappings to map the reads to the annotated genes of the Mt4.0v2 transcriptome (Young et al., 2011; Tang et al., 2014). The resulting count tables were then imported into R (R Core Team, 2018) using the tximport package (v1.4.0; Soneson et al., 2015) and principal component analysis (PCA) and DE analysis at the gene level was performed using DESeq2 (v1.16.1; Love et al., 2014). DESeq2 normalizes the data by fitting a negative binomial GLM with a gene-specific dispersion parameter. Clustering was performed using the Mfuzz package (v2.36; Kumar and Futschik, 2007) using minimum centroid distances as a heuristic measure of appropriate cluster number. Briefly this consisted of compromising between cluster size and number by ascertaining when additional clusters only marginally increased the resolution.

\section{Data processing and visualisation}

All computation and analysis was done on a Macbook Pro (2012; Intel ${ }^{\circledR}$ Core $^{\mathrm{TM}}$ i7-3520M upgraded to 16 Gb RAM) from Apple Inc. (Cupertino, Ca, USA) running Antergos Linux (v17.12; 4.14.11-1-ARCH kernal). Data processing and visualisation was done in $\mathrm{R}$ (R Core Team, 2018) using the Tidyverse suite of packages (v1.2.10; Wickham, 2017) with additional visualisation using the UpsetR package (v1.4; Conway et al., 2017) and the Superheat package (v0.1; Barter, 2018). Analysis and graphs can be reproduced from the accompanying collection of scripts and files in an accompanying figshare repository (see Thomson, 2018).

\section{RESULTS}

It has previously been demonstrated that shifting vernalised $M$. truncatula plants from SD to LD induces flowering and is accompanied by the induction of the expression of FT-like genes MtFTal, MtFTbl and $M t F T b 2$. It was found that three days in LD was sufficient to promote the transition to flowering in $M$. truncatula (but one was insufficient; Laurie et al., 2011). We thus utilised at least three days in LD in our experiments. Here two similar experiments are analysed where plants were grown in SD conditions until they were 10 days old and were then shifted at ZT8 into LD conditions to describe the transcriptomic changes which occur in the M. truncatula leaves following such shifts, alongside the FT-like genes.

In the first experiment sampling of leaves occurred at ZT0 (subjective dawn) and ZT2 when the plants had experienced three days of LD conditions. In the second experiment the plants had experienced five days of LD conditions and sampling occurred at ZT4. With the aim of identifying candidate regulators of FT-like genes these samples capture the constant LD induction of MtFTal and both precede and include the first diurnal peak of MtFTb1 and MtFTb2 at ZT4 in LD (Laurie et al., 2011). These samples (in triplicate) were used to construct RNA-Seq libraries which were subsequently sequenced.

The sequenced RNA-Seq libraries all generated 40-50 million reads with mean quality scores greater than 35 . The quantification of gene abundances reported an average mapping rate of $89.86 \%$ to the 
Mt4.0v2 transcriptome (see Table S1 for full table of abundances in Transcripts per Million; TPM). Thus the data generated are of a high quality and indicates that the Mt4.0v2 transcriptome is reasonably complete.

\section{Differential expression at each time point}

Analysis of this data initially considered pairwise comparisons of gene expression between LD and SD at each timepoint. The significance of any differences observed was assessed using Wald significance tests and since there are three sets of tests, the significance levels of these were adjusted for together using the false discovery rate method.

It was observed that 28,151-29,011 genes had read counts $>1$ (out of the 50,444 in the Mt4.0v2 transcriptome). Of these 6,824 genes at ZT0 (24\% of expressed genes) had statistically different expression $(\alpha=0.05)$. There were 5,523 genes (19\%) at ZT2 and 7,743 genes (25\%) at ZT4 (Table S3). When these lists were filtered for those which had $>2$-fold differences and had $>10$ mean normalised reads then 2,436, 1,309 and 2,661 genes were judged to be DE at ZT0, ZT2 and ZT4 respectively. These numbers are similar to what was observed in an A. thaliana microarray experiment after a SD to LD shift where 2000 genes were DE (Wigge et al., 2005).

The transcript abundances of the LD induced FT-like genes and five other candidate photoperiod pathway genes were assessed (Fig. 1). While absent in SD, large increases in MtFTal transcript abundance were observed in LD at ZT0, ZT2 and ZT4. In addition, appreciable expression of MtFTb1 and MtFTb2 was only seen in LD at ZT4, with minimal to no expression at ZT0 and ZT2 in either SD or LD.
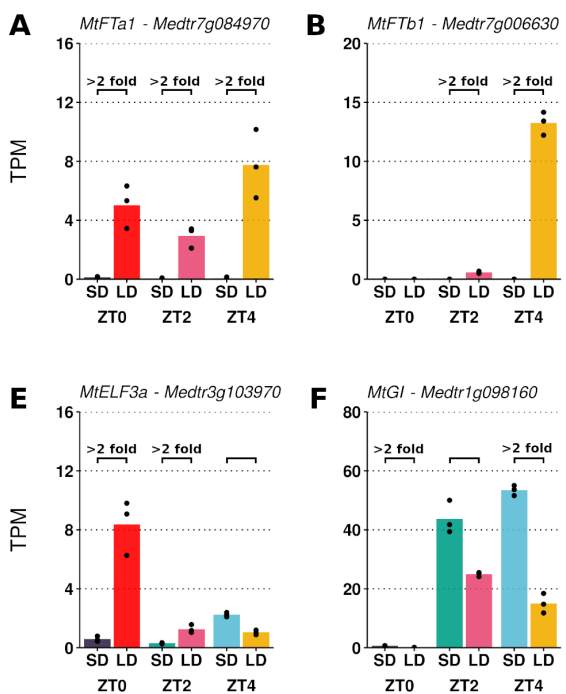

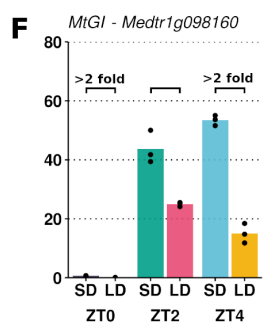

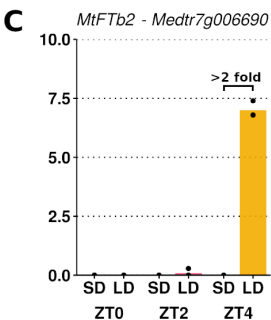

G

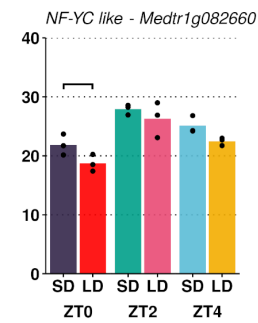

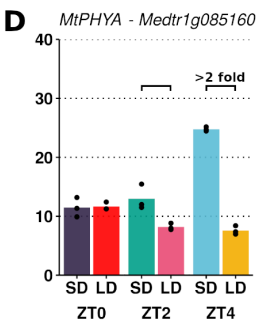

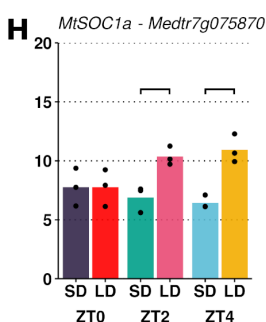

Figure 1. Pairwise comparisons of photoperiod induced changes in expression between LD and SD of a selection of candidate photoperiod pathway genes. Graphs show the mean transcript abundance (in TPM) of eight flowering time genes, both known and hypothesised, across timepoints. Points are the individual replicate libraries. Statistically significant differences $(\alpha=0.05)$ are indicated by the bracket with those differences which show at least a 2 -fold change in transcript abundance annotated.

The differences in expression exhibited by the FT-like genes in Figs. 1a to 1c qualitatively agree with previously reported RT-qPCR time course data where in LD MtFTal has an approximately constant level of expression across the day (Laurie et al., 2011). MtFTb1 and MtFTb2 have been observed to diurnally peak at ZT4 and ZT16 (Laurie et al., 2011), consistent with the DE observed here at ZT4 (Figs. 1b and 1c). This indicates that despite originating from different experiments, these datasets could be analysed together as a ZT0-to-ZT4 time series to observe the pattern of gene expression change following a SD to LD shift.

To further demonstrate that these datasets could be analysed together we considered the diurnal expression of four other genes measured by RT-qPCR in an independent ZT0-ZT20 time series experiment and compared them to our RNA-Seq data (Fig. 2). Here it was observed that in MtFKF1 (Medtr8g105590) 


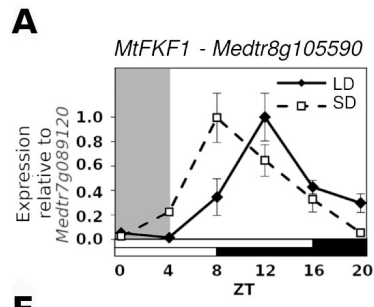

E

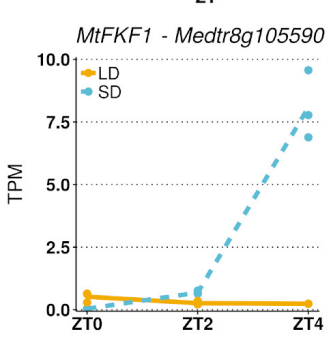

B
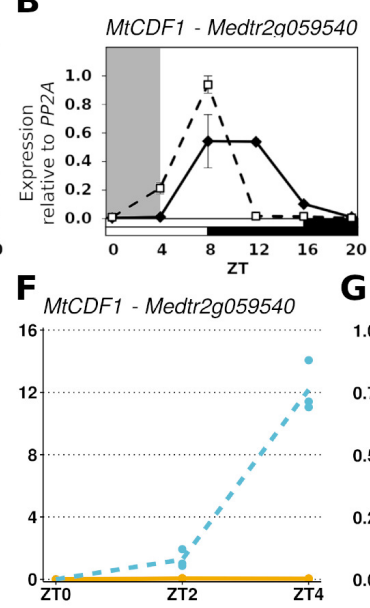

C
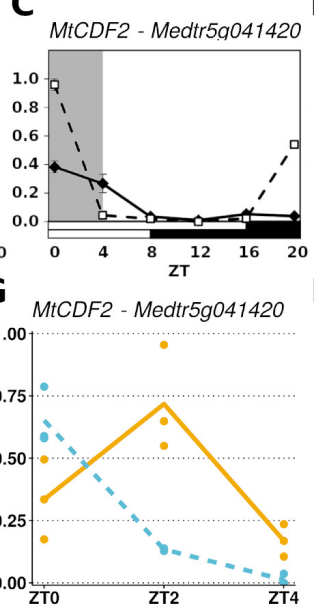

D
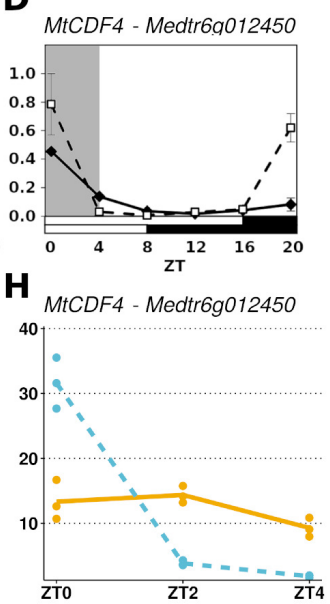

Figure 2. Comparing RT-qPCR and RNA-Seq results from independent experiments to validate combining the RNA-Seq datasets. (A-D) are the diurnal patterns of expression of MtFKF1 (Medtr8g105590), MtCDF1 (Medtr2g059540), MtCDF2 (Medtr5g041420 and MtCDF4 (Medtr6g012450) respectively measured over a diurnal time course using RT-qPCR. The first four hours which overlap with the RNA-Seq data are shaded in grey. Error bars are standard errors of two biological replicates. Samples consist of two fully expanded trifoliate leaves with two biological replicates per time point. For MtFKF1 these samples were pooled and error bars are standard errors of technical replicates (using Medtr7g089120 as a reference gene) while for MtCDF1, MtCDF2 and MtCDF4 these are standard errors of biological replicates (using $P P 2 A$ as a reference gene). (E-H) are the corresponding transcript abundances from the RNA-Seq datasets. The points represent TPM values of the individual replicate libraries plotted with a LOESS smoothed line of best fit for both SD (blue and dotted) and LD (orange and line) samples.

\section{Overview of the time series analysis}

The similarity between the patterns of expression observed in this data with previously reported RTqPCR results for three genes (Figs. 1a to 1c), as well as independently collected RT-qPCR results for an additional four genes (Fig. 2) indicates that there is no significant batch effect which would bias the interpretation of this data as a time series. We then analysed the data in such a manner.

Across the three timepoints 31,363 genes (out of the 50,444) had a read count $>1$ at at least one timepoint (see Fig. S1 for dispersion plot and MA-plot) and were included in the analysis. To take an overarching view of the variation between samples, principal component analysis (PCA) was employed with the first two principal components plotted in Fig. 3. Biological replicates clustered together and 64\% of the observed variation is explained by the first two principal components which strongly align with the time of sampling (PC1) and the photoperiod condition (PC2).

Typically in a pairwise comparison, differences in transcript abundances can be filtered based on fold-change and levels of expression to focus on genes more likely to be biologically consequential. This is difficult in a time series as it is unknown in this instance which timepoint is most relevant to the regulation of FT-like genes. Reflecting on the gene abundances plotted in Fig. 1 it was observed that the genes with differing expression between LD and SD could be broadly grouped into two classes, those which altered their pattern of expression in response to the photoperiodic shift and those which altered the 


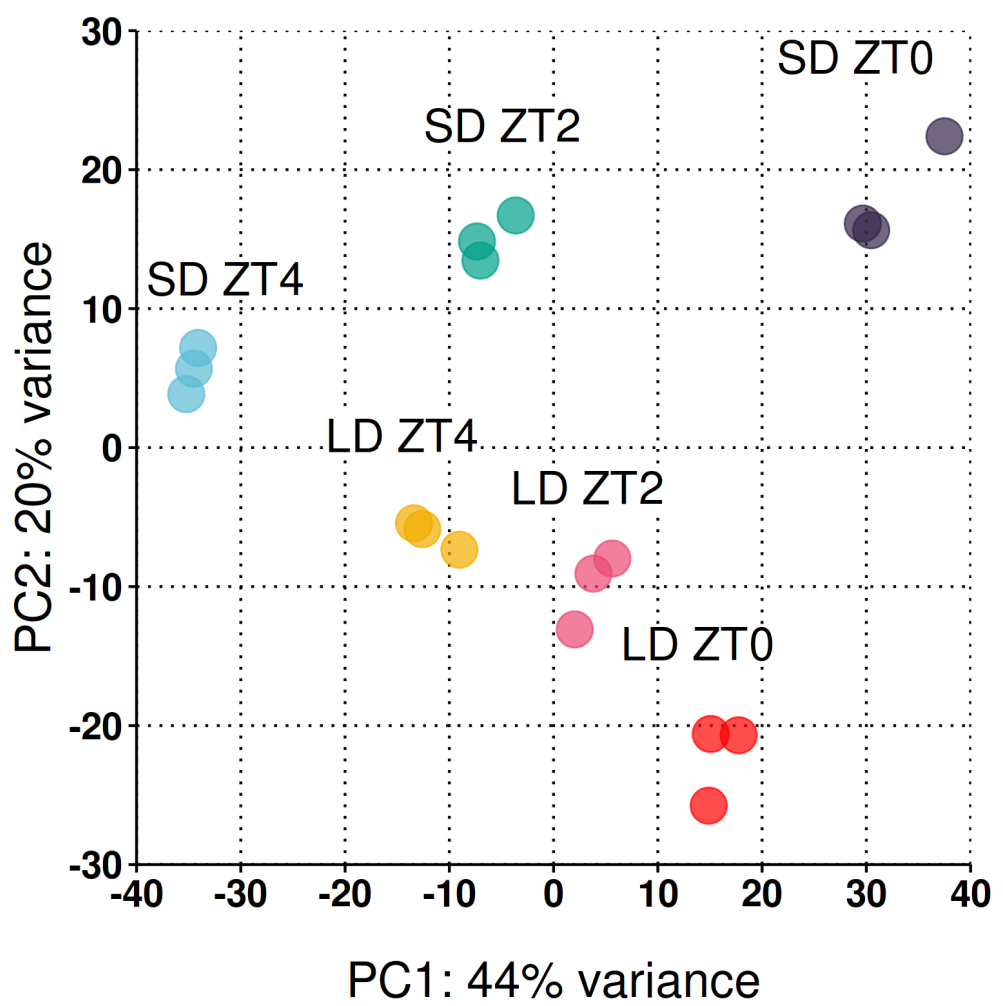

Figure 3. Reduced-space plot of the first two components of a PCA of individual RNA-Seq libraries. This plot gives an overarching perspective on the variation within the dataset with the first two components explaining $64 \%$ of the variation. PC1 strongly aligns with the time of sampling and PC2 the photoperiod condition. The plot was constructed using $\log _{2}$ normalised counts of genes with non-zero read counts.

magnitude of their expression. Specifically, in Fig. 1, the majority of genes (six) change their pattern of expression over time between conditions (MtFTb1, MtFTb2, MtPHYA, MtGI, MtELF3a and MtSOCla), while two genes maintain a similar pattern, but vary in magnitude of expression between conditions (MtFTal and the NF-YC-like gene). It was into these two classes that we decided to segment the time series data followed by clustering.

To classify the genes into these two classes we fit two models to the data. The first model included an interaction term between growth condition (SD or LD) and time of sampling (ZT0, ZT2 and ZT4). This was to identify genes which respond in a condition-specific manner over time such that any changes in the pattern of gene expression brought about by the photoperiodic shift are determined (e.g. MtFTbl in Fig. 1b). Secondly, we repeated the analysis with a model which lacked this interaction term to test for just the effect of condition (LD vs SD) on the magnitude of gene expression (e.g. the NF-YC-like gene in Fig. 1g). This dual approach facilitated the identification of genes which alter their pattern of expression or only the magnitude of their expression respectively.

Alongside this analysis, an a priori list of 146 candidate genes was also assembled consisting of genes shown to, or are suspected of, participating in flowering time regulation via the photoperiod pathway (Tables 1 and 2). In addition to the FT-like genes, the list incorporates photoreceptors, components of the circadian clock and classes of transcription factor which could potentially regulate the FT-like genes chosen based on their role in A. thaliana. These include candidates from the $C D F, T P L$ and $N F-Y$ families as well as additional transcription factors from families with members known to bind the promoter of $F T$ in A. thaliana compiled by Ridge et al. (2016). These include the genes containing CCT-domain and B-box domains as well as the CIB/BEE-like (CBL), CRYPTOCHROME-INTERACTING BASIC-helix-loop-helix (CIB), PHYTOCHROME INTERACTING FACTOR (PIF), and APETALA2 (AP2) families. 


\section{Photoperiod induced changes in the pattern of gene expression}

To identify changes in the pattern of gene expression over time, a generalised linear model was fit using the growth condition and time of sampling as predictors along with an interaction term. A likelihood ratio test was then used to test for genes where this interaction term is significant relative to a reduced model lacking the interaction term. A significant result indicates that the expression of the gene responds in a condition-specific manner over time (i.e. a low p-value indicates a change in expression pattern not solely magnitude). This is illustrated in Fig. 1 where MtPHYA, MtELF3a, MtGI, MtFTb1 and MtSOCla have distinctly different patterns of expression over time in LD compared to SD. On the other hand MtFTal and the NF-YC-like gene Medtrlg082660 show a similar pattern in both LD and SD but at differing magnitudes so in this context are not considered to alter their pattern of expression. While MtFTb2 also appears to alter it's pattern over time, it was not significant in the likelihood ratio test.

This approach resulted in 9,516 genes with altered expression $(\alpha=0.05$; full results in Table S4) or $30.34 \%$ of those tested with $>1$ read. To aid interpretation of these changes and quickly identify the timepoint(s) at which individual genes differed in LD relative to SD, Wald significance tests were used to contrast the difference in expression between the two conditions at each of the three timepoints. The significance levels of these genes were adjusted for all three contrasts together using the false discovery rate method. This resulted in 9,427 of the 9,516 genes having differing expression between LD and SD at a minimum of one timepoint with 6,437, 4,511 and 6,159 for ZT0, ZT2 and ZT4 respectively (Fig. S2a). We consider only genes with $>2$-fold differences with $>10$ mean normalised reads as DE and there were 3,192 genes meeting this criteria at at least one timepoint. This corresponds to 2131, 1062 and 2168 DE genes at ZT0, ZT2 and ZT4 respectively. Full results are listed in Table S5. In these filtered lists of genes $4.9 \%$ of these genes differed in the three timepoints and $32 \%$ differed at two or more timepoints demonstrating how most genes have a single peak, predominantly at ZT0 or ZT4 (Fig. 4a). Notably, there were fewer genes DE at ZT2 than the other timepoints and those that did mostly differed at one or both of the other timpeoints too. Only $27 \%$ of the 1,062 genes DE at ZT2 are unique to the timepoint (compared to $58.4 \%$ for ZT0 and $51.6 \%$ for ZT4).

The interaction term was found to be significant in the majority $(91 / 146 ; 62 \%)$ of the candidate genes associated with photoperiodic regulated flowering including 19/24 (79\%) of the circadian clock and photoreceptor candidate genes and a striking $8 / 8(100 \%)$ of the selected $A P 2$ class of genes (Table 1). Of this set of 91 genes, 90 were statistically different $(\alpha=0.05)$ at one or more timepoints and for 61 genes the difference at at least one timepoint was greater than $>2$-fold difference with $>10$ mean normalised reads. For example, a gene encoding a predicted core component of the core circadian clock, $M t L H Y$, is expressed at $\sim 4$-fold higher levels at ZT0 in SD than LD but this situation is reversed at ZT4 when it is $\sim 7.5$-fold higher in LD than SD. 
A

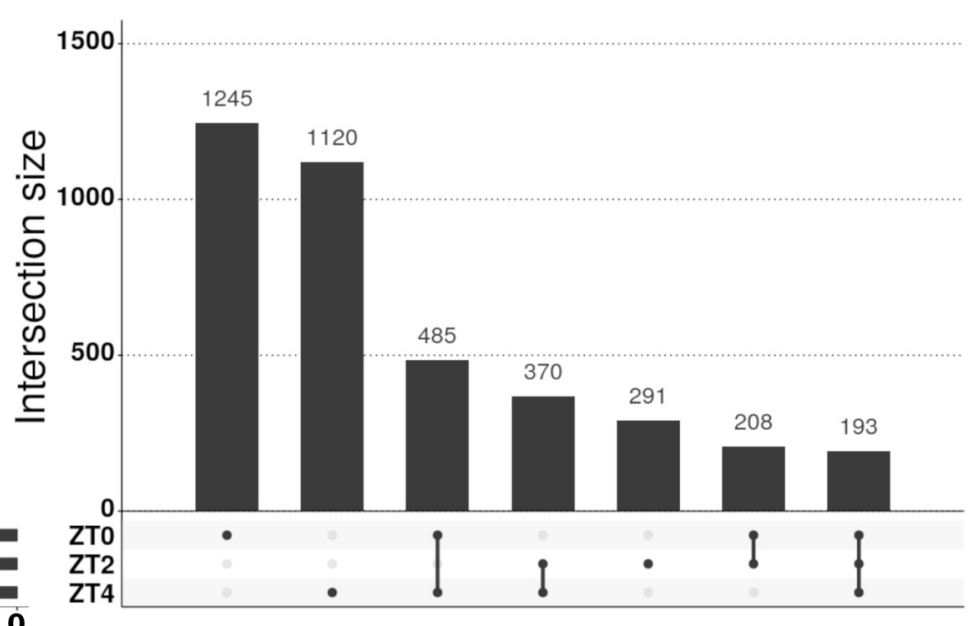

20001000

DE genes per timepoint

B

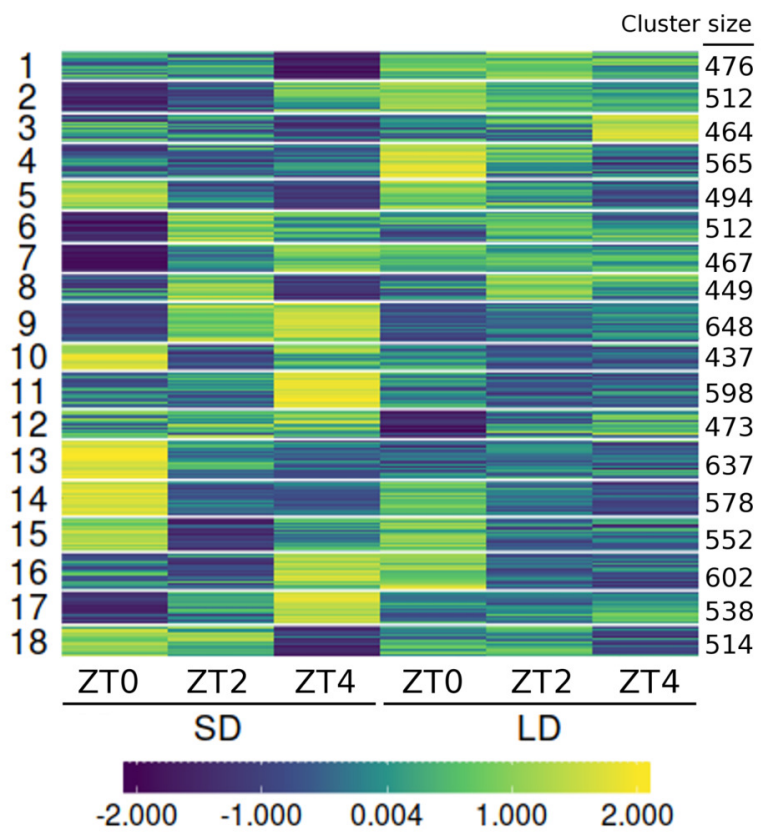

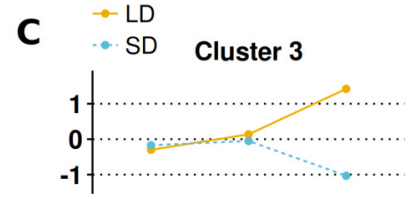

Cluster 9

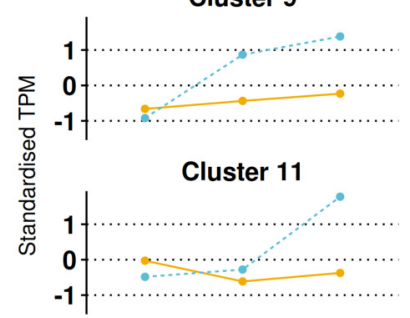

Cluster 17

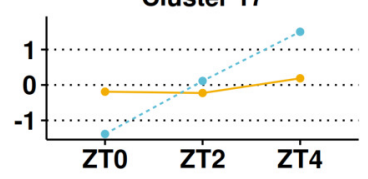

Figure 4. Contrasting timepoint specific expression profiles of the genes which alter their pattern of gene expression in response to the change of photoperiod conditions and clustering the relative changes in expression over time. (A) contrasts the 3,912 DE genes ( $>2$-fold difference and $>10$ mean normalised reads) by the timepoints at which they are DE and plots their overlap. The principal chart plots the size of the overlaps and the supplementary chart presents the number of genes DE (both up or down) in LD relative to SD for each timepoint. (B) depicts a heatmap of standardised gene abundances (such that the average expression value is zero and the standard deviation is one) of all 9,516 genes which alter their pattern of gene expression grouped into 18 clusters using c-means clustering. The number of genes in each cluster is listed alongside. (C) is the mean standardised abundances for selected clusters. Specifically cluster 3 was selected as it contains MtFTbl which characteristically peaks at ZT4 in LD (orange and line) with no expression in SD (blue and dotted). Clusters 9, 11 and 17 were selected as their pattern is opposite to that of cluster 3 in that they peak at ZT4 in SD. Mean standardised abundances for all clusters are plotted in Fig. S5. 
Table 1. Photoperiod induced changes in the pattern of gene expression over time in candidate flowering time loci.

\begin{tabular}{|c|c|c|c|c|c|c|c|c|c|c|c|c|c|c|c|c|c|}
\hline \multirow[b]{2}{*}{ Gene identifier } & \multirow[b]{2}{*}{ Name } & \multirow[b]{2}{*}{ Description (Mt 4.0 annotation) } & \multirow[b]{2}{*}{$\begin{array}{l}\text { Interaction } \\
\text { adj. p-value }\end{array}$} & \multicolumn{4}{|c|}{ ZT0 } & \multicolumn{4}{|c|}{ ZT2 } & \multicolumn{4}{|c|}{ ZT4 } & \multirow[b]{2}{*}{ Cluster } & \multirow[b]{2}{*}{ Membership } \\
\hline & & & & $\begin{array}{c}\text { Mean } \\
\text { read counts }\end{array}$ & $\begin{array}{c}\log _{2} \\
\text { fold-change }\end{array}$ & $\begin{array}{c}\log _{2} \\
\text { s.e }\end{array}$ & $\begin{array}{c}\text { adj. } \\
\text { p-value }\end{array}$ & $\begin{array}{c}\text { Mean } \\
\text { read counts }\end{array}$ & $\begin{array}{c}\log _{2} \\
\text { fold-change }\end{array}$ & $\begin{array}{c}\log _{2} \\
\text { s.e }\end{array}$ & $\begin{array}{c}\text { adj. } \\
\text { p-value }\end{array}$ & $\begin{array}{l}\text { Meam } \\
\text { read counts }\end{array}$ & $\begin{array}{c}\log _{2} \\
\text { fold-change }\end{array}$ & $\begin{array}{c}\log _{2} \\
\text { s.e }\end{array}$ & $\begin{array}{c}\text { adj. } \\
\text { p-value }\end{array}$ & & \\
\hline Medtrlg085160 & MtPHYA & phytochrome protein A & & 1100 & -0.13 & 0.10 & $2.30 \times 10^{-01}$ & 1000 & -0.71 & 0.10 & $5.80 \times 10^{-12}$ & 1900 & -1.60 & 0.10 & $6.90 \times 10^{-60}$ & 11 & 0.41 \\
\hline Medtr2g034040 & MtPHYB & phytochrome protein B & $2.70 \times 10^{-08}$ & 790 & -0.38 & 0.08 & $7.60 \times 10^{-06}$ & 800 & 0.06 & 0.08 & $5.50 \times 10^{-01}$ & 590 & 0.34 & 0.08 & $1.50 \times 10^{-04}$ & 18 & 0.74 \\
\hline Medtr2g049520 & MtPHYE & phytochrome protein & & & & & & & & & & & & & & & \\
\hline Medtr $5 g 063920$ & MtCRYI & cryptochrome protein & $1.70 \times 10^{-13}$ & 5500 & -0.85 & 0.07 & $1.40 \times 10^{-29}$ & 3600 & -0.04 & 0.07 & $6.30 \times 10^{-01}$ & 4700 & -0.26 & 0.07 & $1.00 \times 10^{-03}$ & 10 & 0.53 \\
\hline Medtrlg076190 & $M t C r R Y 2 A$ & cryptochrome 2B apoprotein & $3.60 \times 10^{-21}$ & 2500 & -0.68 & 0.08 & $1.40 \times 10^{-16}$ & 1200 & 0.35 & 0.08 & $8.50 \times 10^{-05}$ & 2000 & -0.65 & 0.08 & $6.30 \times 10^{-15}$ & 15 & 0.47 \\
\hline Medtrlg043190 & $M I C R Y 2 B$ & cryptochrome 2B apoprotein & $4.60 \times 10^{-21}$ & 300 & -0.96 & 0.14 & $8.50 \times 10^{-12}$ & 140 & -1.60 & 0.16 & $7.50 \times 10^{-22}$ & 250 & -3.10 & 0.17 & $1.30 \times 10^{-73}$ & 10 & 0.18 \\
\hline Medtr7g118330 & $M t L H Y$ & late elongated hypocotyl-like protein & $3.00 \times 10^{-70}$ & 37000 & -1.90 & 0.18 & $2.00 \times 10^{-25}$ & 28000 & 0.57 & 0.18 & $2.60 \times 10^{-03}$ & 18000 & 2.90 & 0.18 & $7.40 \times 10^{-58}$ & 18 & 0.27 \\
\hline Medtr4g108880 & MtTOCla & two-component response regulator-like APRR7 protein & $1.50 \times 10^{-58}$ & 75 & 4.40 & 0.36 & $2.10 \times 10^{-31}$ & 63 & 2.00 & 0.23 & $5.10 \times 10^{-17}$ & 120 & -0.97 & 0.18 & $2.60 \times 10^{-07}$ & 2 & 0.69 \\
\hline Medtr3g037390 & MtTOCIb & timing of cab expression 1/PRR response regulator & $2.00 \times 10^{-22}$ & 100 & 1.70 & 0.19 & $4.10 \times 10^{-18}$ & 100 & 0.88 & 0.18 & $\mathbf{2 . 3 0} \times \mathbf{1 0} 0^{-06}$ & 190 & -0.71 & 0.15 & $1.70 \times 10^{-05}$ & 2 & 0.37 \\
\hline Medtr4g061360 & MtPRR37a & PRR response regulator & $1.20 \times 10^{-11}$ & 580 & -4.40 & 0.21 & $1.80 \times 10^{-97}$ & 1600 & -3.40 & 0.18 & $5.30 \times 10^{-77}$ & 4400 & -2.40 & 0.17 & $1.10 \times 10^{-40}$ & 12 & 0.41 \\
\hline Medtrlg067110 & MtPRR37b & two-component response regulator-like APRR7 protein & $1.90 \times 10^{-03}$ & 350 & -2.00 & 0.14 & $3.30 \times 10^{-46}$ & 820 & -1.40 & 0.12 & $5.20 \times 10^{-30}$ & 2700 & -2.10 & 0.12 & $5.60 \times 10^{-66}$ & 9 & 0.34 \\
\hline Medtr3g092780 & MtPRR59a & PRR response regulator & $4.20 \times 10^{-30}$ & 22 & 0.28 & 0.32 & $4.60 \times 10^{-01}$ & 150 & -2.30 & 0.24 & $4.40 \times 10^{-20}$ & 1400 & -4.30 & 0.21 & $4.20 \times 10^{-88}$ & 9 & 0.5 \\
\hline Medtr8g024260 & MtPRR59b & PRR response regulator & $1.40 \times 10^{-05}$ & 54 & -2.30 & 0.24 & $5.20 \times 10^{-21}$ & 210 & -1.20 & 0.15 & $1.50 \times 10^{-14}$ & 1300 & -2.00 & 0.13 & $5.50 \times 10^{-56}$ & 9 & 0.33 \\
\hline Medtr7g118260 & MtPRR $59 \mathrm{c}$ & PRR response regulator & $9.50 \times 10^{-34}$ & 200 & -6.20 & 0.38 & $8.60 \times 10^{-57}$ & 710 & -2.00 & 0.18 & $3.30 \times 10^{-26}$ & 2100 & -1.90 & 0.18 & $4.30 \times 10^{-25}$ & 12 & 0.59 \\
\hline Medtr3g 103970 & MtELF3a & early flowering protein & $1.30 \times 10^{-43}$ & 250 & 3.70 & 0.24 & $7.30 \times 10^{-50}$ & 43 & 2.00 & 0.30 & $1.60 \times 10^{-10}$ & 110 & -1.00 & 0.22 & $2.20 \times 10^{-05}$ & 4 & 0.37 \\
\hline MedtrIg016920 & $M t E L F 3 b$ & EARLY flowering protein, putative & $9.20 \times 10^{-01}$ & & & & & & & & & & & & & & \\
\hline Medtr8g015470 & ELF3-like & hypothetical protein & & & & & & & & & & & & & & te & \\
\hline Medtr8g015480 & ELF3-like & early flowering protein, putative & $4.50 \times 10^{-12}$ & 160 & 1.50 & 0.23 & $4.20 \times 10^{-10}$ & 56 & -0.38 & 0.26 & $2.00 \times 10^{-01}$ & 150 & -0.86 & 0.23 & $4.40 \times 10^{-04}$ & 16 & 0.34 \\
\hline Medtr4g064730 & MtLUXa & myb-like DNA-binding domain, shaqkyf class protein & $1.60 \times 10^{-07}$ & 11 & 3.80 & 0.79 & $6.10 \times 10^{-06}$ & 12 & 0.36 & 0.45 & $5.00 \times 10^{-01}$ & 21 & -0.62 & 0.38 & $1.50 \times 10^{-01}$ & 7 & 0.57 \\
\hline Medtr7g089010 & $M t L U X b$ & MYB-like transcription factor family protein & $2.90 \times 10^{-02}$ & 180 & -1.60 & 0.14 & $6.60 \times 10^{-28}$ & 310 & -1.10 & 0.12 & $4.90 \times 10^{-19}$ & 330 & -1.10 & 0.12 & $7.70 \times 10^{-17}$ & 12 & 0.37 \\
\hline Medtr3g070490 & MtELF4 & early flowering protein & $2.10 \times 10^{-71}$ & 53 & 3.60 & 0.35 & $2.40 \times 10^{-22}$ & 24 & 1.80 & 0.33 & $4.20 \times 10^{-07}$ & 56 & -4.00 & 0.37 & $2.30 \times 10^{-25}$ & 16 & 0.32 \\
\hline Medtr8g020200 & ELF4-like & early flowering protein & $5.80 \times 10^{-01}$ & & & & & & & & & & & & & & \\
\hline Medtr4g 125590 & ELF4-like & early flowering protein & $9.20 \times 10^{-02}$ & - & - & - & & - & - & - & - & - & - & - & - & - & - \\
\hline Medtr2g041310 & ELF4-like & early flowering protein & $2.60 \times 10^{-02}$ & 160 & -1.40 & 0.20 & $8.50 \times 10^{-11}$ & 120 & -0.55 & 0.20 & $1.20 \times 10^{-02}$ & 200 & -0.67 & 0.19 & $1.10 \times 10^{-03}$ & 10 & 0.17 \\
\hline Medtrlg098160 & $M t G I$ & gigantea protein 1B & $2.60 \times 10^{-14}$ & 35 & -3.40 & 0.36 & $5.90 \times 10^{-20}$ & 3300 & -0.86 & 0.12 & $8.40 \times 10^{-12}$ & 4100 & -1.70 & 0.12 & $1.20 \times 10^{-45}$ & 9 & 0.23 \\
\hline Medtr8g105590 & $M t F K F 1$ & flavin-binding kelch repeat F-box protein, putative & $4.60 \times 10^{-56}$ & 14 & 3.30 & 0.60 & $1.50 \times 10^{-07}$ & 24 & -1.40 & 0.33 & $5.40 \times 10^{-05}$ & 250 & -5.00 & 0.29 & $1.70 \times 10^{-64}$ & 11 & 0.78 \\
\hline Medtr2g036510 & MtZTL & galactose oxidase/kelch repeat protein & $6.90 \times 10^{-01}$ & 17 & wo & 0.00 & $1000 \mathrm{~A}=0$ & 27 & 1.70 & 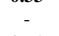 & 然 & wor & 0.00 & (2) & (10) & 11 & 0 \\
\hline Medtr 2 g058520 & MtEI & E1 protein & $2.20 \times 10^{-03}$ & 1.8 & -2.30 & 1.20 & $8.60 \times 10^{-02}$ & 23 & 2.20 & 0.51 & $6.20 \times 10^{-05}$ & 23 & 1.40 & 0.49 & $7.10 \times 10^{-03}$ & 8 & 0.16 \\
\hline Medtr7g084970 & MtFTal & flowering locus protein $\mathrm{T}$ & $5.70 \times 10^{-01}$ & & & & & & & & & & & & & & \\
\hline Medtr7g085020 & MtFTa2 & flowering locus protein $\mathrm{T}$ & $2.80 \times 10^{-03}$ & 17 & 1.00 & 0.44 & $3.80 \times 10^{-02}$ & 11 & -1.10 & 0.49 & $4.90 \times 10^{-02}$ & 28 & -0.97 & 0.39 & $2.30 \times 10^{-02}$ & 11 & 0.3 \\
\hline Medtrog033040 & MtFTa3 & flowering locus protein $\mathrm{T}$ & & & & & & & & & & & & & & & \\
\hline Medtr7g006630 & MtFTbl & flowering locus protein $\mathrm{T}$ & $2.00 \times 10^{-03}$ & 0 & -0.16 & 1.70 & $9.40 \times 10^{-01}$ & 3 & 5.00 & 1.30 & $2.10 \times 10^{-04}$ & 90 & 9.90 & 1.20 & $2.60 \times 10^{-15}$ & 3 & 0.4 \\
\hline Medtr $7 g 006690$ & MtFTb2 & flowering locus protein $\mathrm{T}$ & $6.70 \times 10^{-01}$ & & & & & & & & & & & & & & \\
\hline Medtr2g461760 & MtFULa & MADS-box transcription factor & $0.010-$ & - & - & - & - & - & - & - & - & - & - & - & - & - & - \\
\hline Medtr4g 109830 & MtFULb & MADS-box transcription factor & $3.00 \times 10^{-02}$ & 10 & 1.70 & 0.60 & $8.00 \times 10^{-03}$ & 24 & -0.27 & 0.46 & $6.30 \times 10^{-01}$ & 15 & 1.40 & 0.52 & $1.60 \times 10^{-02}$ & 8 & 0.39 \\
\hline $\begin{array}{l}\text { Medtr7g016630 } \\
\text { Medtr7g075870 }\end{array}$ & $\begin{array}{l}\text { MtFULc } \\
\text { MtSOCla }\end{array}$ & $\begin{array}{l}\text { MADS-box transcription factor } \\
\text { MADS-box transcription factor }\end{array}$ & $7.40 \times 10^{-04}$ & $\therefore$ & -0.16 & $\overline{0 .}$ & $4.60 \times 10^{-01}$ & 120 & 0.53 & 0.18 & $5.30 \times 10^{-03}$ & 150 & 0.86 & 0.17 & $1.90 \times 10^{-06}$ & 3 & 0.35 \\
\hline Medtr8g033250 & MtSOClb & MADS-box transcription fact & & & & & & & $x_{-1}+2$ & & & & 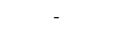 & ${ }^{2}$ & - & 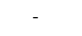 & - \\
\hline Medtr8go33220 & MtSOClc & MADS-box transcription factor & $9.80 \times 10^{-}$ & - & - & - & - & - & - & - & - & - & - & - & - & - & - \\
\hline Medtr2g059540 & $M t C D F l$ & Dof domain zinc finger protein & $8.80 \times 10^{-03}$ & 0 & -0.16 & 1.70 & $9.40 \times 10^{-01}$ & 8.7 & -4.70 & 1.10 & $4.30 \times 10^{-05}$ & 96 & -8.20 & 1.10 & $1.00 \times 10^{-13}$ & 11 & 0.4 \\
\hline Medtr5g041420 & $M t C D F 2$ & DOF zinc finger protein & $1.40 \times 10^{-05}$ & 11 & -1.10 & 0.54 & $5.70 \times 10^{-02}$ & 9.5 & 2.40 & 0.65 & $7.60 \times 10^{-04}$ & 2.7 & 3.80 & 1.30 & $7.30 \times 10^{-03}$ & 5 & 0.19 \\
\hline Medtr5g041530 & $M t C D F 3$ & cycling DOF factor 2 & $3.10 \times 10^{-19}$ & 220 & 0.29 & 0.16 & $9.30 \times 10^{-02}$ & 170 & 1.30 & 0.17 & $8.80 \times 10^{-14}$ & 99 & 2.80 & 0.23 & $1.20 \times 10^{-34}$ & 1 & 0.58 \\
\hline Medtr6g012450 & MtCDF4 & DOF-type zinc finger DNA-binding family protein & $3.40 \times 10^{-77}$ & 860 & -1.40 & 0.14 & $1.60 \times 10^{-21}$ & 350 & 1.90 & 0.16 & $5.40 \times 10^{-31}$ & 270 & 2.40 & 0.17 & $1.30 \times 10^{-45}$ & 5 & 0.34 \\
\hline Medtr6g027460 & MtCDF5 & Dof zinc finger DOF5.2-like protein & $1.40 \times 10^{-18}$ & 370 & 1.40 & 0.19 & $4.40 \times 10^{-13}$ & 350 & 2.00 & 0.19 & $2.30 \times 10^{-24}$ & 100 & 4.90 & 0.37 & $1.30 \times 10^{-37}$ & 1 & 0.69 \\
\hline Medtr7g010950 & MtCDF6 & DOF-type zinc finger DNA-binding family protein & $6.40 \times 10^{-30}$ & 360 & -0.40 & 0.16 & $2.50 \times 10^{-02}$ & 260 & 1.60 & 0.17 & $1.10 \times 10^{-19}$ & 200 & 2.40 & 0.19 & $2.40 \times 10^{-36}$ & 1 & 0.32 \\
\hline
\end{tabular}




\begin{tabular}{|c|c|c|c|c|c|c|c|c|c|c|c|c|c|c|c|c|c|}
\hline \multirow[b]{2}{*}{ Gene identifier } & \multirow[b]{2}{*}{ Name } & \multirow[b]{2}{*}{ Description (Mt4.0 annotation) } & \multirow{2}{*}{$\begin{array}{l}\text { Interaction } \\
\text { adj. p-value }\end{array}$} & \multicolumn{4}{|c|}{ ZT0 } & \multicolumn{4}{|c|}{ ZT2 } & \multicolumn{4}{|c|}{ ZT4 } & \multirow[b]{2}{*}{ Cluster } & \multirow[b]{2}{*}{ Membership } \\
\hline & & & & $\begin{array}{l}\text { Mean } \\
\text { read counts }\end{array}$ & $\begin{array}{l}\log _{2} \\
\text { fold-change }\end{array}$ & $\begin{array}{c}\log _{2} \\
\text { s.e }\end{array}$ & $\begin{array}{c}\text { adj. } \\
\text { p-value }\end{array}$ & $\begin{array}{l}\text { Mean } \\
\text { read counts }\end{array}$ & $\begin{array}{l}\log _{2} \\
\text { fold-change }\end{array}$ & $\begin{array}{c}\log _{2} \\
\text { s.e }\end{array}$ & $\begin{array}{c}\text { adj. } \\
\text { p-value }\end{array}$ & $\begin{array}{l}\text { Meam } \\
\text { read counts }\end{array}$ & $\begin{array}{l}\log _{2} \\
\text { fold-change }\end{array}$ & $\begin{array}{c}\log _{2} \\
\text { s.e }\end{array}$ & $\begin{array}{c}\text { adj. } \\
\text { p-value }\end{array}$ & & \\
\hline Medtr2g016030 & $\begin{array}{l}M t C D F a \\
M t C D F b\end{array}$ & 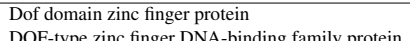 & $8.90 \times 10^{-01}$ & 1400 & & 0.24 & & 800 & & & & 1400 & & & & & \\
\hline Medtr4g082060 & $M t C D F c$ & DOF-type zinc finger DNA-binding family protein & $1.90 \times 10^{-41}$ & 970 & -1.90 & 0.15 & $5.40 \times 10^{-35}$ & 720 & 0.73 & 0.15 & $\mathbf{2 . 8 0 \times 1 0 ^ { - 0 6 }}$ & 760 & $\begin{array}{l}0.33 \\
0.69\end{array}$ & 0.15 & $1.10 \times 10^{-05}$ & 12 & 0.23 \\
\hline Medtr5g041380 & MtCDFd & DOF domain, zinc finger protein & $6.00 \times 10^{-01}$ & - & & & & 120 & & (1.10 & & & & & & & \\
\hline Medtr5g041400 & $\mathrm{MtCDFe}$ & DOF domain, zinc finger protein & $2.70 \times 10^{-06}$ & 8.3 & -2.70 & 0.71 & $2.80 \times 10^{-04}$ & 8.7 & 1.00 & 0.58 & $1.20 \times 10^{-01}$ & 3.5 & 2.50 & 0.98 & $1.70 \times 10^{-02}$ & 18 & 0.21 \\
\hline Medtr6g027450 & $M t C D F f$ & Dof zinc finger DOF5.2-like protein & $3.30 \times 10^{-01}$ & - & - & - & - & - & - & - & - & - & - & - & - & - & - \\
\hline Medtr7g086780 & $M t C D F g$ & Dof zinc finger DOF5.2-like protein & $1.80 \times 10^{-01}$ & - & - & - & - & - & - & - & - & - & - & - & & - & \\
\hline Medtr8g044220 & MtCDFh & DOF-type zinc finger DNA-binding family protein & $1.50 \times 10^{-01}$ & & & & & & & & & & & & & & \\
\hline $\begin{array}{l}\text { Medtr5g085250 } \\
\text { Medtr5g009530 }\end{array}$ & $\begin{array}{l}\text { MtCOPI } \\
\text { SPAI-like }\end{array}$ & $\begin{array}{l}\text { E3 ubiquitin-protein ligase COP1 } \\
\text { ubiquitin ligase cop1, putative }\end{array}$ & 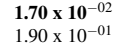 & 1200 & -0.48 & 0.09 & $3.20 \times 10^{-07}$ & 1800 & -0.26 & 0.09 & $6.10 \times 10^{-03}$ & 1600 & -0.08 & 0.09 & $4.50 \times 10^{-01}$ & 8 & 0.17 \\
\hline Medtr8g027985 & SPAT-like & ubiquitin ligase cop1, putative & $8.90 \times 10^{-170}$ & 310 & 3.40 & 0.16 & $5.10 \times 10^{-96}$ & 1000 & -1.10 & 0.11 & $4.10 \times 10^{-20}$ & 2400 & -1.60 & 0.11 & $1.60 \times 10^{-47}$ & 17 & 0.46 \\
\hline Medtr2g085210 & $\begin{array}{l}\text { SPAI-like } \\
\text { TPI lik }\end{array}$ & ubiquitin ligase cop1, putative & $1.30 \times 10^{-01}$ & 1700 & & 012 & $3.30 \times 10^{-13}$ & 1800 & & & $150 \times 10^{-04}$ & & & & & & \\
\hline Medtr2g104140 & $\begin{array}{l}\text { TPL-like } \\
\text { TPLlike }\end{array}$ & topless-like protein & $9.70 \times 10^{-03}$ & 1700 & -0.89 & 0.12 & $3.30 \times 10^{-10}$ & 1800 & -0.48 & 0.12 & $1.50 \times 10^{-04}$ & 1500 & -0.11 & 0.12 & $4.20 \times 10^{-01}$ & 18 & 0.2 \\
\hline $\begin{array}{l}\text { Medtr } 4 \text { g009840 } \\
\text { Medtr4g114980 }\end{array}$ & $\begin{array}{l}\text { TPLLlike } \\
\text { TPLlike }\end{array}$ & $\begin{array}{l}\text { topless-s-ike protein } \\
\text { topless-like protein }\end{array}$ & $\begin{array}{l}6.50 \times 10^{-01} \\
3.50 \times 10^{-01}\end{array}$ & - & - & - & - & - & - & - & - & - & - & - & - & & - \\
\hline $\begin{array}{l}\text { Medtr4g114980 } \\
\text { Medtr2g435370 }\end{array}$ & TPL-like & transducin family protein/WD-40 repeat protein & $2.00 \times 10$ & - & - & - & - & - & - & - & - & - & - & - & - & - & - \\
\hline $\begin{array}{l}\text { Medtrtr243433370 } \\
\text { Medtreg35440 }\end{array}$ & TPL-like & topless-like protein & & - & - & - & & - & - & - & - & - & - & - & - & & - \\
\hline Medtr4gI20900 & TPL-like & topless-like protein & $1.10 \times 10^{-04}$ & 980 & -0.48 & 0.07 & $9.70 \times 10^{-12}$ & 1200 & -0.22 & 0.07 & $1.70 \times 10^{-03}$ & 1400 & -0.05 & 0.06 & $5.40 \times 10^{-01}$ & 12 & 0.34 \\
\hline Medtr7g112460 & TPL-like & topless-like protein & $7.60 \times 10^{-01}$ & - & - & - & - & - & - & & & - & - & - & - & & - \\
\hline Medtr2g065670 & TPL-like & topless-like protein & $8.50 \times 10^{-01}$ & - & - & - & - & - & - & - & - & 烈 & - & - & & - & \\
\hline Medtr2g435380 & TPL-like & topless-like protein & - & - & - & - & - & - & - & - & - & - & - & - & - & - & - \\
\hline Medtrlg012820 & TPL-like & topless-like protein & & - & - & - & - & - & - & - & & - & 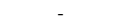 & - & 列 & - & 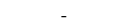 \\
\hline Medtrog444980 & MtFE & myb-like transcription factor family protein & $\mathbf{3 . 0 0 \times 1 0 ^ { - 0 4 }}$ & 430 & 0.46 & 0.10 & $6.50 \times 10^{-06}$ & 380 & 0.03 & 0.10 & $7.90 \times 10^{-01}$ & 440 & 0.61 & 0.10 & $8.50 \times 10^{-10}$ & 1 & 0.29 \\
\hline Medtr3g058980 & NF-YB like & nuclear transcription factor $\mathrm{Y}$ protein & $3.60 \times 10^{-04}$ & 910 & 0.09 & 0.09 & $3.80 \times 10^{-01}$ & 540 & -0.42 & 0.09 & $1.30 \times 10^{-05}$ & 820 & -0.31 & 0.09 & $8.40 \times 10^{-04}$ & 15 & 0.21 \\
\hline Medtr5g095740 & NF-YB like & nuclear transcription factor $\mathrm{Y}$ protein & $\mathbf{2 . 4 0 \times 1 0 ^ { - 0 9 }}$ & 570 & -0.80 & 0.17 & $4.70 \times 10^{-06}$ & 160 & 0.69 & 0.18 & $5.00 \times 10^{-04}$ & 430 & -0.74 & 0.17 & $\mathbf{3 . 3 0} \times \mathbf{1 0} \mathbf{1 0}^{-05}$ & 15 & 0.54 \\
\hline Medtrlg082660 & NF-YC like & nuclear transcription factor $\mathrm{Y}$ protein & $8.80 \times 10^{-02}$ & & & & & & & & & & & & & & \\
\hline Medtr3g099180 & NF-YC like & nuclear transcription factor $\mathrm{Y}$ protein & $5.10 \times 10^{-03}$ & 1800 & 0.51 & 0.12 & $5.40 \times 10^{-05}$ & 1200 & 0.84 & 0.12 & $3.20 \times 10^{-11}$ & 1900 & 0.22 & 0.12 & $9.40 \times 10^{-02}$ & 4 & 0.23 \\
\hline Medtr7g113680 & NF-YClike & nuclear transcription factor Y protein & $9.00 \times 10^{-01}$ & & & & & & & & & & & & & & \\
\hline Medtrlg093600 & MtTEMI & $\mathrm{AP} 2 / \mathrm{ERF}$ and $\mathrm{B} 3$ domain transcription factor & $3.00 \times 10^{-09}$ & 2600 & -1.50 & 0.16 & $3.90 \times 10^{-20}$ & 560 & -0.15 & 0.17 & $4.50 \times 10^{-01}$ & 360 & -1.50 & 0.17 & $1.30 \times 10^{-16}$ & 14 & 0.46 \\
\hline Medtr5g053920 & MtTEM 2 & $\mathrm{AP} 2 / \mathrm{ERF}$ and $\mathrm{B} 3$ domain transcription factor & $7.40 \times 10^{-03}$ & 1900 & -1.20 & 0.17 & $2.10 \times 10^{-11}$ & 1000 & -0.35 & 0.18 & $7.60 \times 10^{-02}$ & 550 & -0.79 & 0.18 & $\mathbf{3 . 3 0} \times \mathbf{1 0} \mathbf{1 0}^{-05}$ & 14 & 0.23 \\
\hline Medtr4g061200 & MtTOEIa & AP2-like ethylene-responsive transcription factor & $1.90 \times 10^{-05}$ & 42 & 1.10 & 0.28 & $1.90 \times 10^{-04}$ & 37 & 0.61 & 0.28 & $4.80 \times 10^{-02}$ & 70 & -0.67 & 0.25 & $1.30 \times 10^{-02}$ & 2 & 0.25 \\
\hline Medtr2g093060 & MtTOEIb & AP2-like ethylene-responsive transcription factor & $1.20 \times 10^{-04}$ & 1200 & -0.15 & 0.08 & $1.00 \times 10^{-01}$ & 1100 & -0.64 & 0.08 & $2.20 \times 10^{-14}$ & 1600 & -0.24 & 0.08 & $4.90 \times 10^{-03}$ & 9 & 0.2 \\
\hline Medtr $7 \mathrm{~g} 100590$ & MtTOEIc & AP2 domain transcription factor & $1.90 \times 10^{-02}, \longrightarrow$ & 49 & 0.82 & 0.27 & $6.00 \times 10^{-03}$ & 28 & 0.30 & 0.30 & $4.00 \times 10^{-01}$ & 64 & -0.36 & 0.26 & $2.10 \times 10^{-01}$ & 16 & 0.38 \\
\hline Medtrlg049140 & MtTOE2 2 & AP2 domain transcription factor & $2.50 \times 10^{-17}$ & 1600 & -1.60 & 0.13 & $4.00 \times 10^{-33}$ & 2500 & -0.65 & 0.13 & $8.40 \times 10^{-07}$ & 2900 & 0.06 & 0.13 & $6.80 \times 10^{-01}$ & 12 & 0.71 \\
\hline Medtr5g016810 & $M t A P 2 a$ & AP2 domain transcription factor & $2.30 \times 10^{-14}$ & 510 & 0.57 & 0.11 & $2.80 \times 10^{-07}$ & 880 & -0.61 & 0.10 & $\mathbf{4 . 3 0} \times \mathbf{1 0} \mathbf{0}^{-09}$ & 730 & -0.24 & 0.10 & $3.30 \times 10^{-02}$ & 6 & 0.46 \\
\hline Medtr4g094868 & $M t A P 2 b$ & AP2 domain transcription factor & $1.50 \times 10^{-12}$ & 260 & 1.00 & 0.13 & $7.80 \times 10^{-14}$ & 390 & -0.17 & 0.12 & $2.30 \times 10^{-01}$ & & -0.24 & 0.13 & $9.70 \times 10^{-02}$ & 6 & 0.21 \\
\hline Medtr7g018170 & MtCOLa & zinc finger constans-like protein & $5.90 \times 10^{-104}>\longrightarrow$ & 4400 & -2.30 & 0.11 & $3.60 \times 10^{-105}$ & 1500 & 0.79 & 0.11 & $1.50 \times 10^{-12}$ & 2200 & 0.38 & 0.11 & $8.10 \times 10^{-04}$ & 13 & 0.36 \\
\hline Medtrlg013450 & $M t C O L b$ & zinc finger constans-like protein & $1.60 \times 10^{-47}$ & 5000 & -0.82 & 0.07 & $8.30 \times 10^{-27}$ & 3800 & 0.16 & 0.08 & $5.50 \times 10^{-02}$ & 3100 & 0.76 & 0.08 & $1.20 \times 10^{-22}$ & 18 & 0.37 \\
\hline Medtr3g105710 & $M t C O L c$ & zinc finger constans-like protein & $1.30 \times 10^{-28}$ & 1700 & -0.48 & 0.05 & $8.30 \times 10^{-22}$ & 860 & 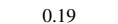 & 0.06 & $1.40 \times 10^{-03}$ & 1200 & 028 & 005 & $5,00 \times 10^{-07}$ & 14 & 0.32 \\
\hline Medtr4g 128930 & MtCOLd & zinc finger constans-like protein & $1.10 \times 10^{-38}$ & 2400 & -2.60 & 0.14 & $6.10 \times 10^{-81}$ & 1500 & -0.34 & 0.14 & $2.00 \times 10^{-02}$ & 2300 & -0.35 & 0.13 & $1.50 \times 10^{-02}$ & 12 & 0.19 \\
\hline Medtr3g082630 & MtCOLe & B-box type zinc finger protein & $1.50 \times 10^{-54}$ & 48 & 7.90 & 1.20 & $3.80 \times 10^{-10}$ & 5 & 2.30 & 0.75 & $5.20 \times 10^{-03}$ & 39 & -3.90 & 0.46 & $4.60 \times 10^{-16}$ & 16 & 0.47 \\
\hline Medtr50069480 & MtCOLf & zinc finger constans-like protein & $9.40 \times 10^{-12}$ & 490 & 4.20 & 0.21 & $1.40 \times 10^{-88}$ & 130 & $2.2>>>>3$ & 0.21 & $4.50 \times 10^{-24}$ & 110 & 2.40 & 0.23 & $4.10 \times 10^{-25}$ & 4 & 0.57 \\
\hline Medtr7g 108150 & $\mathrm{MtCOLg}$ & zinc finger constans-like protei & $6.10 \times 10^{-03}$ & 880 & -0.36 & 0.09 & $\mathbf{7 . 5 0} \times \mathbf{1 0} 0^{-05}$ & 510 & 0.08 & 0.09 & $4.40 \times 10^{-01}$ & 650 & -0.20 & 0.09 & $3.60 \times 10^{-02}$ & 14 & 0.38 \\
\hline Medtr7g083540 & MtCOLh & zinc finger constans-like protein & $6.20 \times 10^{-07}$ & 57 & 0.14 & 0.24 & $6.30 \times 10^{-01}$ & 69 & -0.19 & 0.23 & $5.00 \times 10^{-01}$ & 61 & -1.70 & 0.26 & $8.40 \times 10^{-11}$ & 16 & 0.1 \\
\hline Medtr8g104190 & $\mathrm{MtCOLi}$ & zinc finger constans-like protein & $5.50 \times 10^{-02}$ & & & & & 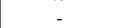 & & & & & & & & & \\
\hline Medtr2g088900 & $M t C O L j$ & zinc finger constans-like protein & $2.50 \times 10^{-02}$ & 79 & -0.81 & 0.29 & $1.10 \times 10^{-02}$ & 83 & 0.28 & 0.29 & $4.00 \times 10^{-01}$ & 75 & 0.30 & 0.29 & $3.80 \times 10^{-01}$ & 18 & 0.28 \\
\hline Medtrlg110870 & MtCOLk & zinc finger constans-like protein & $1.40 \times 10^{-19}$ & 2000 & 1.70 & 0.16 & $2.20 \times 10^{-22}$ & 230 & -0.05 & 0.18 & $8.20 \times 10^{-01}$ & 1200 & -0.53 & 0.17 & $3.10 \times 10^{-03}$ & 15 & 0.19 \\
\hline$g 032240$ & $M t C M F 1$ & CCT motif protein & $7.90 \times 10^{-04}$ & 910 & -0.26 & 0.08 & $2.10 \times 10^{-03}$ & 470 & 0.11 & 0.09 & $2.50 \times 10^{-01}$ & 1100 & 0.16 & 0.08 & $5.40 \times 10^{-02}$ & 15 & 0.34 \\
\hline Medtr4gI27420 & $M t C M F 2$ & import apparatus protein & $2.40 \times 10^{-19}$ & 180 & 0.35 & 0.13 & $1.60 \times 10^{-02}$ & 660 & $\begin{array}{l}-0.78 \\
-0.78\end{array}$ & 0.11 & $6.00 \times 10^{-12}$ & 840 & -1.30 & 0.11 & $\mathbf{3 . 5 0} \times \mathbf{1 0}^{-31}$ & 9 & 0.43 \\
\hline Medtr5g072780 & $M t C M F 3$ & CCT motif protein & $8.60 \times 10^{-02}$ & & & & - & & & - & - & & & & & - & \\
\hline Medtr3g 100040 & MtCMF5 & GATA transcription factor & $0 \times 10^{-01}$ & - & - & - & - & - & - & - & - & - & - & - & - & - & - \\
\hline Medtr3g100050 & $M t C M I$ & GATA transcription factor & $6.80 \times 10^{-01}$ & - & - & - & - & - & - & - & - & - & - & - & - & - & - \\
\hline Medtr5g066510 & MtCMF7 & GATA transcription factor & $7.30 \times 10^{-01}$ & - & - & - & - & - & - & - & - & - & - & - & - & - & - \\
\hline Medtr4g093730 & $M t C M F 8$ & GATA transcription factor & $4.30 \times 10^{-01}$ & - & - & - & - & - & - & - & - & - & - & - & - & - & - \\
\hline
\end{tabular}




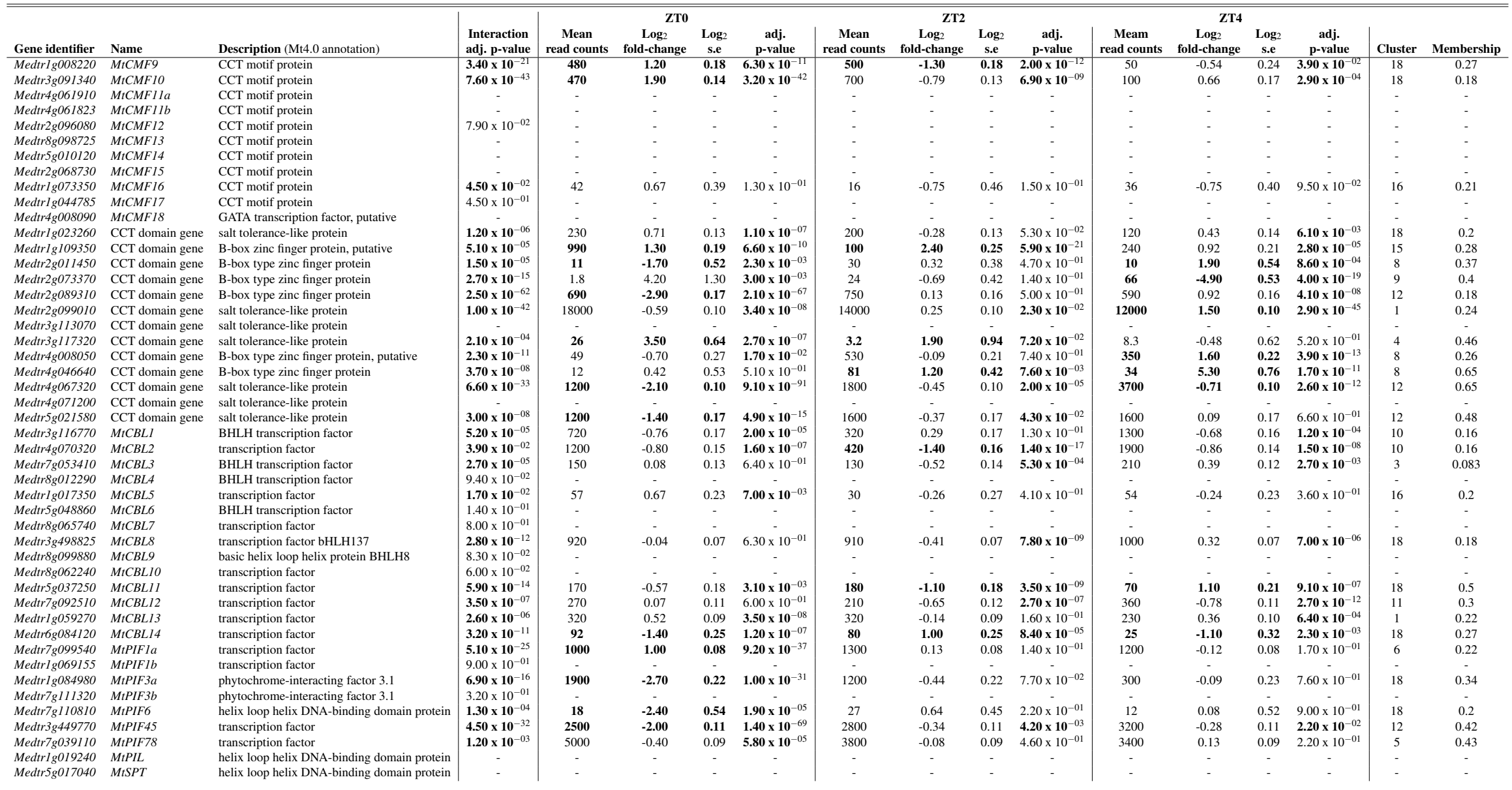

The genes listed in this table are loci known or hypothesised to participate in the photoperiod pathway in legumes along with homologues of the core components of the pathway in A. thaliana.

They include potential $F T$ promoter binding genes compiled by Ridge et al. (2016) from which the naming of MITOEIa to $M I S P T$ derives.
Table depicts the the adjusted p-value for the interaction between time and condition. Note that if the adjusted p-value is significant each

ast between conditions at timepoints ZT0, ZT2 and ZT4 is also given to facilitate 
To get a broader view of the results, the mean abundances of all 9,516 genes which altered their pattern of expression were taken and $\log _{2}$ transformed, before being standardised to have a mean of zero and standard deviation of one. The standardised mean abundances were then clustered into 18 clusters using c-means clustering (tbl:S6; see Fig. S3a for cluster number optimisation) and visualised in Fig. 4b. This algorithm assigns a membership score (between 0 and 1 ) for each gene to each cluster describing the degree to which an individual observation belongs to a given cluster (see Fig. S4 for distribution of membership scores). A gene is then assigned to the cluster for which it has the highest membership. Cluster 3, which has 464 genes in it, was of particular interest as MtFTbl is present. Thus these genes have patterns of expression similar to MtFTb1 and may be involved in regulating MtFTb1 or involved in similar processes. Clusters 9, 11 and 17 (648, 598 and 538 genes respectively) are also of interest as they contain genes with an opposite expression pattern to cluster 3, some of which may thus be negative regulators of MtFTb1 (e.g. MtFTa2 is in cluster 11). Specifically, these three clusters have peaks of expression at ZT4 in SD which are not present in LDs (Fig. 4c).

With regard to candidate photoperiodic flowering time genes, alongside $M t F T b 1$ in cluster 3 there were only two genes from Table 1. These were MtSOCla (Medtr7g075870) and a BHLH transcription factor gene called MtCBL3 (Medtr7g053410). MtSOCla is a downstream target of MtFTal (Medtr7g084970) and demonstrated to affect flowering time (Jaudal et al., 2018). Another cluster 3 candidate flowering related gene Medtr3g101520 encodes a B3 domain, as does E1, the most important locus in the photoperiod pathway of the tropical legume soybean (Glycine $\max$ (L.) Merr.; Xia et al., 2012). On the other hand, the predicted circadian clock-like genes PRR37a, $b$ and PRR59a-c (Matsushika et al., 2000; Nakamichi et al., 2005) all have profiles opposite to that of $M t F T b 1$ with three included in cluster 9 (Table 1). Additionally Medtrlg033620, a SHAQKYF class MYB-like DNA-binding domain gene, is present in cluster 11 which is interesting as proteins of this class have recently been implicated in the regulation of $F T$ in A. thaliana (e.g. EFM and FE; Yan et al., 2014; Abe et al., 2015).

Next, genes in each cluster were then ranked by their fold-change at ZT4 because MtFTb1 has its greatest difference in expression between the two conditions at this timepoint (962-fold up in LD; Fig. 1b). We focused on transcription factor genes and observed significant fold changes in transcript levels of genes encoding a number of zinc finger proteins. For instance, in cluster 3 at ZT4, the zinc finger (Ran-binding) family gene Medtr4g113840 was 45-fold elevated in LD compared to SD and the DOF-type zinc finger DNA-binding family gene Medtr3g091820 was increased 2-fold. In contrast, in cluster 9 the B-box type zinc finger protein Medtr2g073370 was 29-fold more abundant in SD than in LD at ZT4 and in cluster 11, Medtr2g059540, also a DOF domain zinc finger gene (denoted MtCDF1 in Table 1) was 300-fold higher in SD than in LD. Furthermore in cluster 17, the zinc finger (Ran-binding) family gene Medtr6g069400 is 7-fold elevated in SD compared to LD at ZT4.

\section{Changes in the magnitude of gene expression}

There is a class of genes which change their level of expression in response to the shift from SD to LD conditions, but this does not alter the relative changes which occur at differing timepoints. Thus it is only the magnitude, not the pattern of expression which changes (e.g. MtFTal or the NF-YC-like gene Medtrlg082660 in Figs. 1a and 1g). To identify these genes a simpler model was fit the data which lacked the interaction term between growth condition and time of sampling.

It was observed that in this model 8,695 genes differed in the magnitude of their expression between conditions ( $\alpha=0.05$; Table S7), but this was reduced to 4,694 when those that also altered the pattern of their expression were omitted. Therefore $14.96 \%$ of genes with $>1$ read $(4,694 / 31,363$ genes) alter just the magnitude of their expression in response to the shift in photoperiod conditions. To provide timepoint level resolution of these changes Wald significance tests were again used to contrast the 4,694 genes (Table S8) with the significance levels of these genes adjusted for all three contrasts together using the false discovery rate method. This resulted in 4,161 of the 4,694 genes differing in magnitude at least one timepoint with 2,715, 2,268 and 2,666 for ZT0, ZT2 and ZT4 respectively (Fig. S2b). When considering genes with $>2$ fold differences and $>10$ mean normalised reads as DE, only 819 genes differed in magnitude at at least one timepoint and at ZT0, ZT2 and ZT4 this corresponded to 457, 353 and 454 genes respectively. Here it was observed that only 65 genes (7.9\%) of this set of genes are consistently higher in LDs than SDs while 54 genes (9.99\%) are consistently lower (Fig. 5a). The numbers in these classes go up to 187 genes (22.8\%) and 139 genes (17\%) respectively when genes differing at two or more timepoints are included. Like the class of genes which altered their pattern of expression there are fewer DE genes at ZT2. 
Results for the candidate photoperiod loci are summarised in Table 2. Specifically these are the candidate photoperiodic flowering time genes which were not classed as changing their pattern of expression between conditions. Here 15/55 (27\%) of the genes are classed as altering just their magnitude in response to the photoperiodic shift. However only 9/15 (60\%) have statistically different levels of expression at two or more timepoints and looking down the list MtFTal is the only gene to consistently differ $>2$-fold with $>10$ mean normalised reads. Thus none of these candidate photoperiod genes are expressed similarly to MtFTal which shows $>30$-fold higher levels in LD than in SD at all timepoints.

We then assessed the sets of genes whose expression is either consistently higher or lower in LDs than SDs (65 and 54 genes respectively) for other candidate genes which could potentially play a role in the regulation of flowering time. These include Medtr8g091720 a NF-Y-like gene, which exhibits consistently greater expression (2.5-5.6-fold) in LD than in SD, like that of MtFTal. Genes which consistently show reduced expression in LD compared to SD include Medtr2g014200 which encodes a squamosa promoter-binding-like protein as well as genes associated with sugar transport. For instance, Medtr3g074180 encoding a trehalose-6-phosphate phosphatase is 2-3 fold lower accross the three timepoints and Medtr0204s0040 a sugar porter family MFS transporter which not expressed in LD at all. Sugar transport is a process linked to flowering time and the regulation of $F T$ in A. thaliana (Wahl et al., 2013).

In addition, while not previously linked to flowering time, these lists also contain a number of genes which likely have regulatory functions such as Medtr5g079220 encoding a R2R3-MYB transcription factor, Medtr3g107940 which produces a FBD protein and Medtr8g012655 which is the gene for an ethylene response factor. These genes all have greater expression in LD compared to SD. Conversely, genes which have reduced expression in LD compared to SD include Medtr7g012790 encoding a circadian clock coupling factor ZGT, Medtr7g 105780 encoding an ovate transcriptional repressor, Medtr3g031220 which produces a WRKY transcription factor and Medtr8g026960 which is the gene for a homeobox associated leucine zipper protein.

Relaxing the criteria for differential expression slightly and reconsidering the candidate photoperiod loci also suggests that MtCDFf (Medtr6g027450) could be investigated further as it is higher in LD in all three timepoints (8.57-fold, 3.48-fold and 12.99-fold respectively) however its expression is overall quite low with an average of only 2.7 normalised reads at ZT4. Other potential candidates on the list include the ELF4-like gene Medtr8g020200, consistently 1.8-2.3-fold higher in SD compared to LD, as is MtCMF17 (Medtrlg044785) although, like MtCDFf, the expression in these datasets is low. This may reflect that these genes are cell-type specific and so only expressed in a fraction of those sampled. 
A

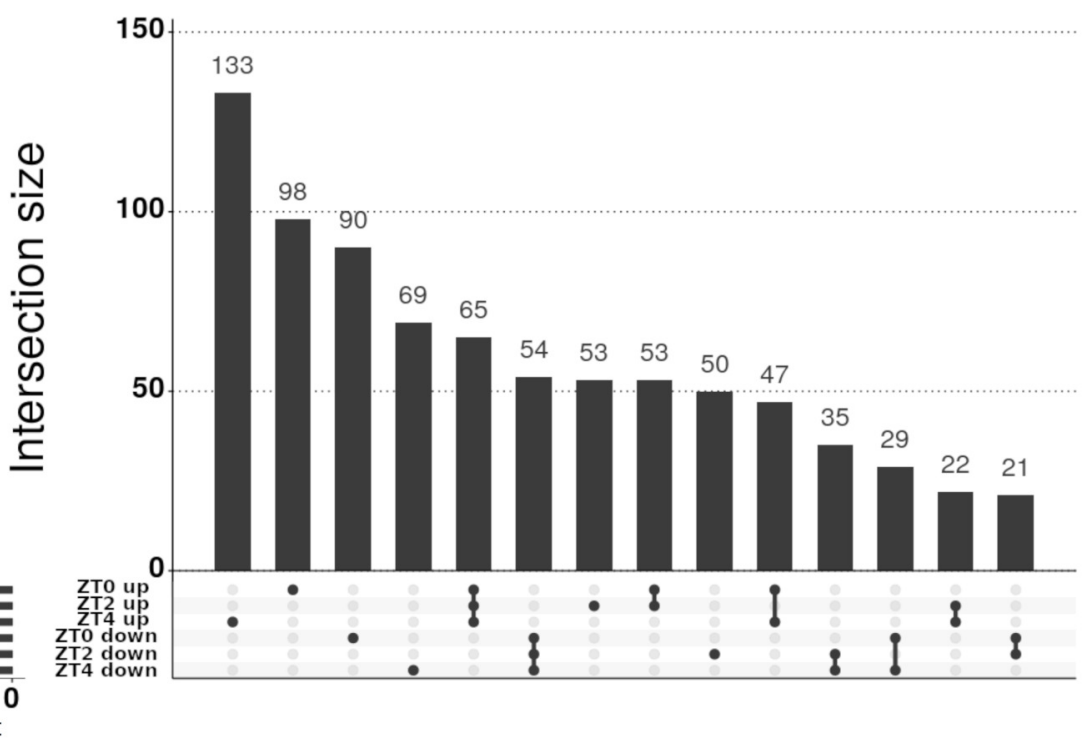

B

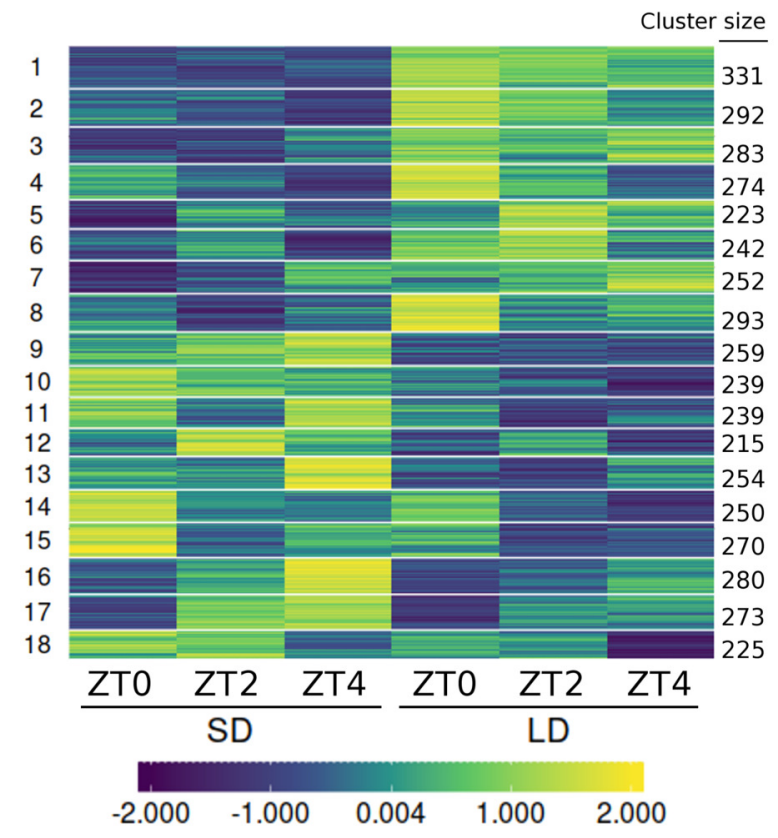

C $\quad \rightarrow$ LD

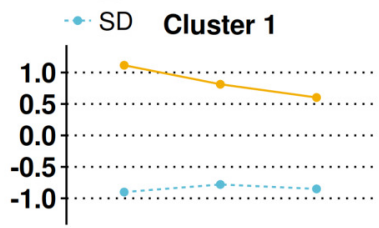

Cluster 9

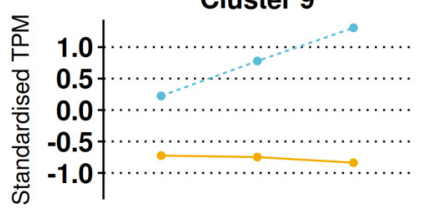

Cluster 10

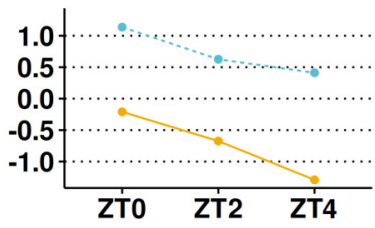

Figure 5. Contrasting timepoint specific expression profiles of the genes which alter only the magnitude of their gene expression in response to the change of photoperiod conditions and clustering the relative changes in expression over time. (A) contrasts the direction of the 819 genes which alter just the magnitude of their gene expression and are DE ( $>2$-fold difference and $>10$ mean normalised reads) at one or more timepoint. The principal chart plots the size of the overlaps between timepoints and the supplementary chart presents the number of genes DE (either up or down) in LD relative to SD for each timepoint. Membership within each group for individual genes, including non DE genes, is given in Table S8. (B) is the standardised and clustered abundances (such that the average expression value is zero and the standard deviation is one) of all 4,694 genes which were classed as just altering the magnitude of their gene expression in response to the photoperiod shift clustered into 18 clusters using c-means clustering. The number of genes in each cluster is listed alongside. (C) is the mean standardised abundance for clusters examined in more detail are plotted with cluster 1 selected for containing MtFTal which is expressed in LD at all timepoints but not SD and clusters 9 and 10 were selected as genes in these clusters are consistently higher in SD (blue and dotted) than in LD (orange and line). Mean standardised abundances for all clusters are plotted in Fig. S6. 
Table 2. Photoperiod induced changes in the magnitude of gene expression in candidate flowering time loci not observed to alter their pattern over time (see Table 1).

\begin{tabular}{|c|c|c|c|c|c|c|c|c|c|c|c|c|c|c|c|c|c|}
\hline Gene identifier & Name & Description (Mt4.0 annotation) & $\begin{array}{c}\begin{array}{c}\text { Condition } \\
\text { adj. p-value }\end{array} \\
\end{array}$ & $\begin{array}{c}\text { Mean } \\
\text { read count }\end{array}$ & $\begin{array}{c}\text { ZT0 } \\
\text { Log } \\
\text { fold-change }\end{array}$ & $\begin{array}{c}\log _{2} \\
\text { s.e }\end{array}$ & $\begin{array}{c}\text { adj. } \\
\text { p-value }\end{array}$ & $\begin{array}{c}\mathrm{ZT2} \\
\text { Mean } \\
\text { read count }\end{array}$ & $\begin{array}{c}\log _{2} \\
\text { fold-change }\end{array}$ & $\begin{array}{c}\log _{2} \\
\text { s.e. }\end{array}$ & $\begin{array}{c}\text { ZT4 } \\
\text { adj. } \\
\text { p-value }\end{array}$ & $\begin{array}{c}\text { Mean } \\
\text { read count }\end{array}$ & $\begin{array}{c}\log _{2} \\
\text { fold-change }\end{array}$ & $\begin{array}{c}\log _{2} \\
\text { s.e. }\end{array}$ & $\begin{array}{c}\text { adj. } \\
\text { p-value }\end{array}$ & Cluster & Membership \\
\hline Medtr2g049520 & MIPHYE & phytochrome protein & & 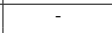 & - & - & - & - & - & - & - & - & - & - & 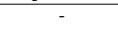 & - & \\
\hline Medtrlg016920 & MtELF3b & EARLY flowering protein, putative & $5.50 \times 10^{-01}$ & - & - & - & - & - & - & $\cdot$ & - & - & - & - & - & - & - \\
\hline $\begin{array}{l}\text { Meddrtrg0154700 } \\
\text { Metdrog20200 }\end{array}$ & $\begin{array}{l}\text { ELL3-like } \\
\text { ELF4-like }\end{array}$ & $\begin{array}{l}\text { hypothetical protein } \\
\text { early flowering protein }\end{array}$ & $1.60 \times 10^{-20}$ & 100 & -0.88 & 0.18 & $1.50 \times 10^{-05}$ & 98 & -1.2 & 0.19 & $7.40 \times 10^{-09}$ & 210 & -0.94 & 0.16 & $1.70 \times 10^{-07}$ & 13 & 0.43 \\
\hline Medtr4gI25590 & ELF4-like & early flowering protein & $1.60 \times 10^{-01}$ & - & -0.0 & 0.10 & $100 \mathrm{x}-$ & & - & - & $1.00 \times-$ & - & - & & & - & 0.4 \\
\hline Medtr2g036510 & MIZTL & galactose oxidase/kelch repeat protein & $8.20 \times 10^{-01}$ & & & & & 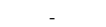 & 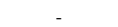 & 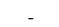 & 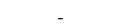 & - & & & - & - & - \\
\hline Medtr7g084970 & MtFTal & flowering locus protein $T$ & $1.80 \times 10^{-27}$ & 28 & 5.2 & 0.79 & $1.20 \times 10^{-09}$ & 16 & 6.4 & 1.2 & $1.90 \times 10^{-06}$ & 55 & 6.8 & 0.89 & $2.10 \times 10^{-12}$ & 1 & 0.32 \\
\hline Medtr6g033040 & MtFTa3 & flowering locus protein $\mathrm{T}$ & & & & - & 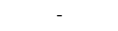 & & & & & & ( & & & & 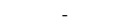 \\
\hline Medtr $7 g 006690$ & MtFTb2 & flowering locus protein $\mathrm{T}$ & $5.20 \times 10^{-01}$ & - & - & - & - & - & - & - & - & - & - & - & - & - & - \\
\hline Medtr2g461760 & MtFULa & MADS-box transcription factor & $2.90 \times 10^{-01}$ & - & - & - & - & & - & - & - & - & - & - & - & - & - \\
\hline Medtr $7 \mathrm{~g} 016630$ & MtFULc & MADS-box transcription factor & & - & - & - & - & - & - & - & - & - & - & - & - & - & - \\
\hline Medtr8g033250 & $M t S O C l b$ & MADS-box transcription factor & & - & - & - & - & - & - & - & - & - & - & - & - & - & - \\
\hline Medtr 80033220 & MtSOCIc & MADS-box transcription factor & $9.80 \times 10^{-01}$ & - & - & - & - & - & - & - & - & - & - & - & - & - & - \\
\hline Medtr2g016030 & $M t C D F a$ & Dof domain zinc finger protein & $7.60 \times 10^{-01}$ & - & - & - & - & - & - & - & - & - & - & - & - & - & - \\
\hline Medtr5g041380 & MtCDFd & DOF domain, zinc finger protein & $4.40 \times 10^{-01}$ & & & & & & & & & & & & & & \\
\hline Medtr6g027450 & MtCDFf & Dof zinc finger DOF5.2-like protein & $2.10 \times 10^{-09}$ & 12 & 3.1 & 0.67 & $4.10 \times 10^{-05}$ & 9.8 & 1.8 & 0.57 & $5.50 \times 10^{-03}$ & 2.7 & 3.7 & 1.3 & $9.70 \times 10^{-03}$ & 2 & 0.53 \\
\hline $\begin{array}{l}\text { Medtr7 } 7086780 \\
\text { Medtrog } 0420\end{array}$ & $\begin{array}{l}M t C D F g \\
M t C D F h\end{array}$ & $\begin{array}{l}\text { Dof zinc finger DOF5,2-2-1ke protein } \\
\text { DOF-tye zinc finger DNA-binding family protein }\end{array}$ & $\begin{array}{l}5.00 \times 10^{-02} \\
\mathbf{9 . 5 0} \times 10^{-05}\end{array}$ & 65 & & 021 & & 60 & & & & 63 & & & & & \\
\hline $\begin{array}{l}\text { Meeterrgo0442L0 } \\
\text { Medtr } 5 \text { g009530 }\end{array}$ & SPAI-like & 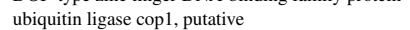 & $\begin{array}{l}1.50 \times 10^{-06} \\
1.90 \times 10^{-06}\end{array}$ & $\begin{array}{l}63 \\
890\end{array}$ & $\begin{array}{l}0.93 \\
-0.22\end{array}$ & $\begin{array}{c}0.21 \\
0.062\end{array}$ & $\begin{array}{l}1.10 \times 10^{-04} \\
1.40 \times 10^{-03}\end{array}$ & $\begin{array}{l}60 \\
980\end{array}$ & $\begin{array}{c}0.49 \\
-0.28\end{array}$ & $\begin{array}{c}0.21 \\
0.061\end{array}$ & $\begin{array}{l}4.00 \times 10^{-02} \\
3.50 \times 10^{-05}\end{array}$ & $\begin{array}{l}63 \\
850\end{array}$ & $\begin{array}{c}0.26 \\
-0.096\end{array}$ & 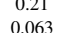 & $2.80 \times 10^{-01}$ & ${ }_{18}^{2}$ & $\begin{array}{l}0.4 \\
0.52\end{array}$ \\
\hline Medtr2g085210 & SPAl-like & ubiquitin ligase cop1, putative & $2.40 \times 10^{-03}$ & 4100 & & 0.086 & $4.30 \times 10^{-01}$ & 4600 & 0.36 & 0.086 & $1.80 \times 10^{-04}$ & 4700 & 0.18 & 0.086 & $6.20 \times 10^{-02}$ & 6 & 0.45 \\
\hline Medtr4g009840 & TPL-like & topless-like protein & $9.00 \times 10^{-11}$ & 3200 & -0.35 & 0.11 & $3.30 \times 10^{-03}$ & 3000 & -0.51 & 0.11 & $1.40 \times 10^{-05}$ & 2000 & -0.49 & 0.11 & $4.30 \times 10^{-05}$ & 18 & 0.88 \\
\hline Medtr4g114980 & TPL-like & topless-like protein & $1.20 \times 10^{-05}$ & 840 & -0.25 & 0.14 & $9.90 \times 10^{-02}$ & 960 & -0.42 & 0.13 & $6.30 \times 10^{-03}$ & 1200 & -0.59 & 0.13 & $8.90 \times 10^{-05}$ & 9 & 0.57 \\
\hline Medtr2g435370 & TPL-like & transducin family protein/WD-40 repeat protein & & - & - & & & & - & & & & & & & & \\
\hline Medtr2g435440 & TPL-like & topless-like protein & & 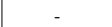 & 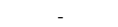 & - & - & 列 & & - & & - & - & - & & - & - \\
\hline Medtr7g112460 & TPL-like & topless-like protein & $1.60 \times 10^{-18}$ & 570 & -0.57 & 0.12 & $1.20 \times 10^{-05}$ & 430 & -0.65 & 0.12 & $1.40 \times 10^{-06}$ & 450 & -0.73 & 0.12 & $3.40 \times 10^{-08}$ & 10 & 0.5 \\
\hline Medtr2g065670 & TPL-like & topless-like protein & $6.70 \times 10^{-01}$ & - & - & - & - & & - & - & - & - & - & ${ }^{2}$ & - & & - \\
\hline Medtr2g435380 & TPL-like & topless-like protein & & - & - & - & - & - & - & - & - & - & - & - & - & - & - \\
\hline Medtrlg012820 & TPL-like & topless-like protein & & & & & & & & & & & & & & & \\
\hline Medtrlg082660 & NF-YC like & nuclear transcription factor $\mathrm{Y}$ protein & $6.40 \times 10^{-03}$ & 370 & -0.38 & 0.094 & $2.90 \times 10^{-04}$ & 490 & -0.14 & 0.089 & $1.60 \times 10^{-01}$ & 530 & -0.065 & 0.088 & $5.10 \times 10^{-01}$ & 12 & 0.34 \\
\hline Medtr 7 g1 13680 & NF-YC like & nuclear transcription factor $\mathrm{Y}$ protein & $1.10 \times 10^{-10}$ & 490 & 0.37 & 0.1 & $1.40 \times 10^{-03}$ & 380 & 0.46 & 0.11 & $1.40 \times 10^{-04}$ & 400 & 0.41 & 0.11 & $6.30 \times 10^{-04}$ & 4 & 0.69 \\
\hline Medtr8g104190 & $\mathrm{MtCOLi}$ & zinc finger constans-like protein & $2.00 \times 10^{-02}$ & 5800 & -0.032 & 0.07 & $6.90 \times 10^{-01}$ & 4100 & -0.092 & 0.071 & $2.50 \times 10^{-01}$ & 6100 & -0.29 & 0.07 & $1.80 \times 10^{-04}$ & 15 & 0.28 \\
\hline Medtr5g072780 & MtCMF3 & CCT motif protein & $6.10 \times 10^{-01}$ & & & & & & & & & & & & & & \\
\hline Medtr3g100040 & $M t C M F 5$ & GATA transcription factor & $4.50 \times 10^{-02}$ & 240 & -0.4 & 0.13 & $8.00 \times 10^{-03}$ & 180 & -0.14 & 0.14 & $3.80 \times 10^{-01}$ & 210 & -0.063 & 0.14 & $6.80 \times 10^{-01}$ & 14 & 0.83 \\
\hline Medtr3g100050 & MtCMF6 & GATA transcription factor & $1.20 \times 10^{-01}$ & & & & & & & & & & - & & & & - \\
\hline Medtrsg066510 & $M t C M F 7$ & GATA transcription factor & $2.40 \times 10^{-01}$ & - & - & - & - & - & - & - & - & - & - & - & - & - & - \\
\hline Medtr4g093730 & MtCMF8 & GATA transcription factor & $1.70 \times 10^{-01}$ & - & - & - & - & - & - & - & - & - & - & - & - & - & - \\
\hline Medtr4g061910 & MtCMFIIa & CCT motif protein & - & - & - & - & - & - & - & - & - & - & - & - & - & - & - \\
\hline Medtr4g061823 & MtCMFIlb & CCT motif protein & - & - & - & - & - & - & - & - & - & - & - & - & - & - & - \\
\hline Medtr2g096080 & MtCMFI2 & CCT motif protein & $1.50 \times 10^{-01}$ & - & - & - & - & 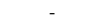 & - & - & 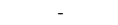 & - & - & - & 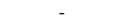 & - & - \\
\hline Medtr $8 g 098725$ & MtCMFI3 & CCT motif protein & & - & - & - & - & & - & - & - & - & - & - & - & - & - \\
\hline Medtrsg010120 & MtCMFI4 & CCT motif protein & - & - & - & - & - & - & - & - & - & - & & - & - & - & - \\
\hline Medtr2g068730 & MtCMFI5 & CCT motif protein & - & - & - & - & - & - & - & - & - & - & - & - & - & - & - \\
\hline Medtrlg044785 & MtCMFI7 & CCT motif protein & $1.80 \times 10^{-02}$ & 2.3 & -2 & 1 & $8.70 \times 10^{-02}$ & 4.5 & -1.6 & 0.76 & $6.80 \times 10^{-02}$ & 13 & -0.51 & 0.54 & $4.00 \times 10^{-01}$ & 16 & 0.54 \\
\hline Medtr4g008090 & MtCMFI8 & GATA transcription factor, putative & & & & & & & & & & & - & & & & \\
\hline Medtr3g113070 & CCT domain gene & salt tolerance-like protein & - & - & - & - & - & - & - & - & - & - & - & - & - & - & - \\
\hline Medtr $4 g 071200$ & CCT domain gene & salt tolerance-like protein & & - & - & - & - & - & - & - & - & - & - & - & - & - & - \\
\hline Medtr8g012290 & MtCBLA & BHLH transcription factor & $1.40 \times 10^{-01}$ & - & - & - & - & - & - & - & - & - & - & - & - & - & - \\
\hline Medtr $5 g 048860$ & MtCBL6 & BHLH transcription factor & $4.20 \times 10^{-01}$ & - & - & - & - & $-\theta^{-1}$ & - & - & - & - & - & - & $-\sigma^{-1}$ & - & - \\
\hline Medtr8g065740 & $M t C B L 7$ & transcription factor & $6.20 \times 10^{-01}$ & - & - & - & - & - & - & - & - & - & - & - & - & - & - \\
\hline Medtr $8 g 099880$ & MtCBL9 & basic helix loop helix protein BHLH8 & $5.40 \times 10^{-01}$ & - & & - & & & & - & & & & & & - & \\
\hline Medtr8g062240 & MtCBLIO & transcription factor & $8.90 \times 10^{-03}$ & 110 & 0.066 & 0.16 & $7.20 \times 10^{-01}$ & 64 & 0.28 & 0.19 & $1.90 \times 10^{-01}$ & 82 & 0.72 & 0.18 & $3.60 \times 10^{-04}$ & 4 & 0.31 \\
\hline Medtrlg069155 & MtPIFIb & transcription factor & $9.30 \times 10^{-01}$ & & & & & & & & & & & & & & \\
\hline Medtr7g111320 & MtPIF3b & phytochrome-interacting factor 3.1 & $5.80 \times 10^{-01}$ & - & - & - & - & - & - & - & - & - & - & - & - & - & - \\
\hline Medtrlg019240 & MtPIL & helix loop helix DNA-binding domain protein & & - & - & - & - & - & - & - & - & - & - & - & - & - & - \\
\hline Medtr $5 g 017040$ & MtSPT & helix loop helix DNA-binding domain protein & - & & & - & & & - & - & & - & & - & & - & \\
\hline
\end{tabular}

The genes listed in this table are loci known or hypothesised to participate in the photoperiod pathway in legumes along with homologues of the core components of the pathway in A. thaliana.

They include potential $F T$ promoter binding genes compiled by Ridge et al. (2016) from which the naming of $M t C O L i$ to $M t S P T$ derives.

Table depicts the the adjusted p-value for the interaction between time and condition. Note that if the adjusted p-value is significant each contrast between conditions at timepoints ZT0, ZT2 and ZT4 is also given to facilitate

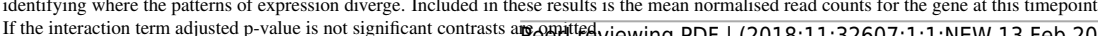

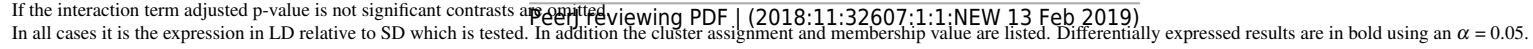


All 4,694 genes which differed in the magnitude of their expression between conditions were then clustered into 18 clusters (Fig. 5b, Fig. S3b and Table S9). MtFTal was present in cluster 1 which had 331 genes. However, none of the selected photoperiodic candidate genes clustered with MtFTal. Clusters 9 (259 genes) and 10 (239 genes) have patterns opposite to that of cluster 1 (Fig. 5c). A TPL-like gene from Table 2 is in cluster 9 and a second TPL-like gene is in cluster 10. In addition, in clusters 9 and 10, a number of other flowering time candidates are present including Medtr0020s0120 in cluster 9, which is similar to the FT antagonist TERMINAL FLOWER 1 in A. thaliana (Jaeger et al., 2013; Wickland and Hanzawa, 2015). Also present in cluster 9 are a trio of genes encoding B3 binding domain proteins Medtrlg021410, Medtrlg021435 and Medtrlg021500 and pair of genes which encode SHAQKYF class MYB transcription factors Medtr0036s0260 and Medtr5g027550. These genes are notable for genes containing these domains have been associated with flowering time, as has the jumonji domain protein encoding gene Medtr2g011630 in cluster 10 (Xia et al., 2012; Abe et al., 2015; Yan et al., 2014).

A total of 35/65 of the consistently differentially expressed genes which are higher in LDs than SDs (Fig. 5a) are present in cluster 1. These include Medtrlg099440 which encodes a membrane-associated kinase regulator-like protein, Medtr6g086805 a heat shock transcription factor gene and Medtr 40009110 which encodes a helix loop helix DNA-binding domain protein. Similarly, clusters 9 and 10 contain 19/54 of the consistently differentially expressed genes which are lower in LDs than SDs. These genes include the ethylene response factor gene Medtr5g016750 and Medtr4g119422 encoding a cullin-like protein.

We then ranked the clustered genes by their fold-change between LD and SD at ZTO. Strikingly, in cluster 1, within the top 13 genes with the largest fold increase in LD compared to SD at ZT0, MtFTal is ranked 8th, while 10 of the other genes were homologues of IRON MAN (IMA)/FE-UPTAKE-INDUCING PEPTIDE 1 (FEPI) genes. These encode mobile signalling peptides integral for the uptake of of iron from the soil and octuple imalfepl mutants in A. thaliana result in severe chlorosis (Grillet et al., 2018; Hirayama et al., 2018). They were also identified in a recent reannotation of the M. truncatula genome to identify small, secreted peptides (de Bang et al., 2017). In addition cluster 3, which is similar to cluster 1 , has the remaining annotated IMA/FEP1 genes as 4 of the top 5 genes with the largest fold-change differences at ZTO.

\section{DISCUSSION}

This study presents a thorough overview of the changes in the M. truncatula leaf tissue transcriptome following a shift of vernalised plants from SD to LD conditions between ZT0 and ZT4. Our data are of very high quality with an average mapping rate of $89.86 \%$. This suggests that while the existing Mt4.0v2 transcriptome captures the majority of the signal in the data there is nevertheless space to improve it. To date, a majority of transcriptomic datasets in M. truncatula have been generated from root tissue, not leaves (e.g. experiments in the MtGEA database are $>50 \%$ root tissue (Benedito et al., 2008)). Thus this dataset broadens the understanding of gene expression in the aerial tissue of M. truncatula, in particular in differing photoperiodic conditions. Consequently it could be incorporated into future cross-species comparisons with datasets like that of Wu et al. (2014) who performed a similar shift experiment in the SD-responsive soybean.

While the data presented here consist of two composite datasets, and so interpretation requires caution, the results of this analysis are nevertheless biologically plausible. Initial pairwise comparisons of gene expression between LD and SD at each timepoint individually qualitatively agreed with previously published expression profiles of MtFTa1, MtFTb1 and MtFTb2 (Figs. 1a to 1c; Laurie et al., 2011). Furthermore, independent RT-qPCR timecourses of $M t F K F 1, M t C D F 1, M t C D F 2$ and $M t C D F 4$ are similar to the transcript abundances seen in these RNA-Seq datasets (Fig. 2). From these results it was concluded that there is no significant batch effect between datasets and that it is appropriate to interpret this data as a time series.

The dual approaches taken to analyse the data as a time series first considered the interaction between condition and time and then just the effect of the condition. This successfully identified the genes which alter the pattern or just the magnitude of their expression respectively. This approach could serve as a template for similar datasets in other plants which lack a CO-like regulator. Given the significant role the circadian clock plays in the regulation of the A. thaliana transcriptome (Covington et al., 2008; Michael et al., 2008) and the manner in which the photoperiod regulates the circadian clock (Nohales and Kay, 2016), it is unsurprising to see that a greater number of genes were classed as altering their pattern of expression $(9,516 / 31,363$ of detectable genes; $30.34 \%)$ than only the magnitude of their expression 
$(4,694 / 31,363 ; 14.96 \%)$. This is especially true of our selected candidate photoperiodic genes (Table 1) where $62 \%$ had a significant change in pattern.

Clustering was employed to subset the two classes of DE genes further based on their normalised abundances across the three timepoints. The low membership scores reveal the small degree of separation between clusters. This may be a feature of gene expression data, but the strength of c-means clustering is that it allows the certainty of cluster assignment to be assessed (Tables S6 and S9). An alternative approach to subset the classes of genes would be to group them based on functional gene set descriptors such as Gene Ontology (GO) terms and cluster until individual clusters become enriched for single GO terms. However since in M. truncatula only 37\% of genes have annotated GO terms (Tang et al., 2014), their use is currently of limited utility.

This study has identified additional candidate photoperiodic flowering time genes for future characterising and reverse genetics screens. In terms of identifying genes co-expressed with the LD-induced M. truncatula FT-like genes, candidate genes such as the zinc finger gene Medtr4g113840 and the B3 domain transcription factor gene Medtr3g101520 (both in the same cluster as MtFTb1) present as future avenues of inquiry as potential regulators of photoperiodic flowering. Conversely, it would be interesting to investigate the CCT containing B-box type zinc finger gene Medtr2g073370 which has the opposite pattern of expression to MtFTbl consistent with a repressive role. Finally, it is notable that none of the list of candidate photoperiod genes responded in a similar way to the SD to LD photoperiodic shift as the potent floral activator MtFTal (Table 2). However a number of other potential regulators were identified, such as the NF-Y-like Medtr8g091720 which has consistently higher expression in LD or the pair of TPL-like genes (Medtr4g114980 and Medtr7g112460) and ethylene response factor gene Medtr5g016750 which all have a pattern of expression opposite to MtFTal.

The experiments analysed in this study focused on the first four hours of the diurnal cycle where, in LD, MtFTal is induced and which precede and include the first peak in expression of LD induced MtFTbl and MtFTb2 at ZT4 (Laurie et al., 2011). It should be noted that the clustering presented in this study is limited to the timepoints sampled and cannot be considered predictive of the pattern in which genes are expressed later in the day. Genes in the same cluster may have divergent patterns later in the day. Given the diurnal pattern of expression of MtFTb1 and MtFTb2 (Laurie et al., 2011), our ability to identify candidate regulators which share patterns of expression would be enhanced by the inclusion of additional samples from later timepoints. Notably at ZT8 to capture the trough and ZT16 to capture the second peak in expression of MtFTb1 and MtFTb2 in LD. This is because samples from later timepoints would facilitate greater discrimination between expressed genes and thus result in smaller clusters. However, it is also possible that the regulator of M. truncatula FT-like gene expression is post-translationally regulated because light/protein dependent mechanisms are common in photoperiodic and circadian regulatory networks. In this case, it might not be possible to identify the regulators using a co-expression approach.

\section{CONCLUSIONS}

This study further elucidates the photoperiodic acceleration of flowering in the reference legume species M. truncatula which interestingly appears to lack a CO-like regulator. We found that the photoperiodic shift from SD to LD conditions had a large effect on the leaf transcriptome with 14,210 genes altering their pattern or magnitude of expression. Candidate regulators that were co-expressed with the LD-induced FT-like genes were identified by clustering. It was notable that none of the list of candidate photoperiod genes responded to the photoperiodic shift in a similar manner as that of the potent floral activator MtFTal, and few were similar to that of the MtFTb genes. Thus this analysis further supports the idea that FT-like genes in $M$. truncatula are uncoupled from the photoperiodic transcriptional networks seen in other species and that flowering time in M. truncatula is induced in a novel manner. Future work will focus on molecular-genetic analysis of the function of the candidate regulators identified in this study in $M$. truncatula photoperiodic flowering.

\section{ACKNOWLEDGMENTS}

We would like to thank our anonymous reviewers, Nicole Cloonan, Peter Tsai, Kevin Chang and William Schierding for their advice and insight in analyzing the data. Many thanks also to the other members of the Plant Molecular Biology Lab at the University of Auckland, especially Betty Phan and Lulu Zhang. 


\section{REFERENCES}

Abe, M., Kaya, H., Watanabe-Taneda, A., Shibuta, M., Yamaguchi, A., Sakamoto, T., Kurata, T., Ausin, I., Araki, T., and Alonso-Blanco, C. (2015). FE, a phloem-specific MYB-related protein, promotes flowering through transcriptional activation of FLOWERING LOCUS T and FLOWERING LOCUS T INTERACTING PROTEIN 1. The Plant Journal, 83(6):1059-1068.

Andrés, F. and Coupland, G. (2012). The genetic basis of flowering responses to seasonal cues. Nature Reviews. Genetics, 13:627-639.

Ballerini, E. S. and Kramer, E. M. (2011). In the light of evolution: a reevaluation of conservation in the $C O-F T$ regulon and its role in photoperiodic regulation of flowering time. Frontiers in Plant Science, 2(81).

Barter, R. (2018). Superheat: An R package for generating beautiful and customizable heatmaps. GitHub repository: https://github.com/rlbarter/superheat.

Benedito, V. A., Torres-Jerez, I., Murray, J. D., Andriankaja, A., Allen, S., Kakar, K., Wandrey, M., Verdier, J., Zuber, H., Ott, T., Moreau, S., Niebel, A., Frickey, T., Weiller, G., He, J., Dai, X., Zhao, P. X., Tang, Y., and Udvardi, M. K. (2008). A gene expression atlas of the model legume Medicago truncatula. The Plant Journal, 55(3):504-513.

Bushnell, B. (2018). Bbtools: A suite of fast, multithreaded bioinformatics tools designed for analysis of dna and rna sequence data. Joint Genome Institute: https://jgi.doe.gov/ data-and-tools/bbtools/.

Cheng, X., Li, G., Tang, Y., and Wen, J. (2018). Dissection of genetic regulation of compound inflorescence development in Medicago truncatula. Development, 145:dev.158766.

Conway, J. R., Lex, A., and Gehlenborg, N. (2017). UpSetR: An R package for the visualization of intersecting sets and their properties. Bioinformatics, 33(18):2938-2940.

Covington, M. F., Maloof, J. N., Straume, M., Kay, S. A., and Harmer, S. L. (2008). Global transcriptome analysis reveals circadian regulation of key pathways in plant growth and development. Genome Biology, 9(8):R130.

Dally, N., Xiao, K., Holtgräwe, D., and Jung, C. (2014). The B2 flowering time locus of beet encodes a zinc finger transcription factor. PNAS, 111(28):10365-10370.

de Bang, T., Lundquist, P. K., Dai, X., Boschiero, C., Zhuang, Z., Pant, P., Torres-Jerez, I., Roy, S., Nogales, J., Veerappan, V., Dickstein, R., Udvardi, M. K., Zhao, P. X., and Scheible, W. (2017). Genome-wide Identification of Medicago Peptides involved in Macronutrient Responses and Nodulation. Plant Physiology, 175:1669-1689.

Fudge, J. B., Lee, R. H., Laurie, R. E., Mysore, K. S., Wen, J., Weller, J. L., and Macknight, R. C. (2018). Medicago truncatula $S O C 1$ genes are up-regulated by environmental cues that promote flowering. Frontiers in Plant Science, 9:496.

Gibeaut, D. M., Hulett, J., Cramer, R., and Seemann, J. R. (1997). Maximal biomass of Arabidopsis thaliana using a simple, low-maintenance hydroponic method and favorable environmental conditions. Plant Physiology, 115:317-319.

Gnesutta, N., Kumimoto, R. W., Swain, S., and Chiara, M. (2017). CONSTANS imparts DNA sequencespecificity to the histone-fold NF- YB / NF-YC dimer. The Plant Cell, 29(6):1516-1532.

Goralogia, G., Liu, T., Zhao, L., Panipinto, P., Groover, E., Bains, Y., and Imaizumi, T. (2017). CYCLING DOF FACTOR 1 represses transcription through the TOPLESS co-repressor to control photoperiodic flowering in Arabidopsis. The Plant Journal, 92(2):244-262.

Grillet, L., Lan, P., Li, W., Mokkapati, G., and Schmidt, W. (2018). IRON MAN, a ubiquitous family of peptides that control iron transport in plants. Nature Plants, [Epub ahead of print].

Hayama, R., Yokoi, S., Tamaki, S., Yano, M., and Shimamoto, K. (2003). Adaptation of photoperiodic control pathways produces short-day flowering in rice. Nature, 422(6933):719-722.

Hecht, V., Knowles, C. L., Schoor, J. K. V., Liew, L. C., Jones, S. E., Lambert, M. J. M., and Weller, J. L. (2007). Pea LATE BLOOMER1 is a GIGANTEA ortholog with roles in photoperiodic flowering, deetiolation, and transcriptional regulation of circadian clock. Plant Physiology, 144:648-661.

Hecht, V., Laurie, R. E., Vander Schoor, J. K., Ridge, S., Knowles, C. L., Liew, L. C., Sussmilch, F. C., Murfet, I. C., Macknight, R. C., and Weller, J. L. (2011). The pea GIGAS gene is a FLOWERING LOCUS T homolog necessary for graft-transmissible specification of flowering but not for responsiveness to photoperiod. The Plant Cell, 23:147-161.

Highkin, H. R. (1956). Vernalisation in Peas. Plant Physiology, 31(5):399-403. 
Hill, J. R. (2000). Jester - Application No: 98/201. Plant Varieties Journal, 13(2):40.

Hirayama, T., Lei, G. J., Yamaji, N., Nakagawa, N., and Ma, J. F. (2018). The Putative Peptide Gene FEP1 Regulates Iron Deficiency Response in Arabidopsis. Plant and Cell Physiology, Corrected Proof. Jaeger, K. E., Pullen, N., Lamzin, S., Morris, R. J., and Wigge, P. A. (2013). Interlocking feedback loops govern the dynamic behavior of the floral transition in Arabidopsis. The Plant Cell, 25:820-833.

Jaudal, M., Zhang, L., Che, C., Li, G., Tang, Y., Wen, J., Mysore, K. S., and Putterill, J. (2018). A SOC1-like gene MtSOC1a promotes flowering and primary stem elongation in Medicago. Journal of Experimental Botany, 69(20):4867-4880.

Kakar, K., Wandrey, M., Czechowski, T., Gaertner, T., Scheible, W.-R., Stitt, M., Torres-Jerez, I., Xiao, Y., Redman, J. C., Wu, H. C., Cheung, F., Town, C. D., and Udvardi, M. K. (2008). A community resource for high-throughput quantitative RT-PCR analysis of transcription factor gene expression in Medicago truncatula. Plant Methods, 4:18.

Kumar, L. and Futschik, M. (2007). Mfuzz: A software package for soft clustering of microarray data Bioinformation. Bioinformation, 2(1):5-7.

Laurie, R. E., Diwadkar, P., Jaudal, M., Zhang, L., Hecht, V., Wen, J., Tadege, M., Mysore, K. S., Putterill, J., Weller, J. L., and Macknight, R. C. (2011). The Medicago FLOWERING LOCUS T homolog, MtFTal, is a key regulator of flowering time. Plant Physiology, 156(4):2207-2224.

Li, C., Distelfeld, A., Comis, A., Dubcovsky, J., and Osmond, G. (2011). Wheat flowering repressor VRN2 and promoter CO2 compete for interactions with NUCLEAR FACTOR-Y complexes. The Plant Journal, 67:763-773.

Liew, L. C., Hecht, V., Laurie, R. E., Knowles, C. L., Vander Schoor, J. K., Macknight, R. C., and Weller, J. L. (2009). DIE NEUTRALIS and LATE BLOOMER 1 contribute to regulation of the pea circadian clock. The Plant Cell, 21(10):3198-3211.

Livak, K. J. and Schmittgen, T. D. (2001). Analysis of relative gene expression data using real-time quantitative PCR and the $2^{-\Delta \Delta C t}$ Method. Methods, 25(4):402-408.

Love, M. I., Huber, W., and Anders, S. (2014). Moderated estimation of fold change and dispersion for RNA-Seq data with DESeq2. Genome Biology, 15:550.

Matsushika, a., Makino, S., Kojima, M., and Mizuno, T. (2000). Circadian waves of expression of the APRR1/TOCl family of pseudo-response regulators in Arabidopsis thaliana: insight into the plant circadian clock. Plant \& Cell Physiology, 41(9).

Michael, T. P., Mockler, T. C., Breton, G., McEntee, C., Byer, A., Trout, J. D., Hazen, S. P., Shen, R., Priest, H. D., Sullivan, C. M., Givan, S. a., Yanovsky, M., Hong, F., Kay, S. a., and Chory, J. (2008). Network discovery pipeline elucidates conserved time-of-day-specific cis-regulatory modules. PLoS genetics, 4(2):e14.

Nakamichi, N., Kita, M., Ito, S., Yamashino, T., and Mizuno, T. (2005). PSEUDO-RESPONSE REGULATORS, PRR 9, PRR7 and PRR5, Together play essential roles close to the circadian clock of Arabidopsis thaliana. Plant and Cell Physiology, 46(5):686-698.

Nohales, M. A. and Kay, S. A. (2016). Molecular mechanisms at the core of the plant circadian oscillator. Nature Structural and Molecular Biology, 23(12):1061-1069.

Patro, R., Duggal, G., Love, M. I., Irizarry, R. A., and Kingsford, C. (2017). Salmon provides fast and bias-aware quantification of transcript expression. Nature Methods, 14(4):417-419.

Pearce, S., Shaw, L. M., Lin, H., Cotter, J. D., Li, C., Dubcovsky, J., Hughes, H., Chase, C., Maryland, C. L., Moore, B., Alto, P., and California, J. D. (2017). Night-break experiments shed light on the Photoperiodl-mediated flowering. Plant Physiology, 174:1139-1150.

Pin, P. A., Zhang, W., Vogt, S. H., Dally, N., Büttner, B., Schulze-Buxloh, G., Jelly, N. S., Chia, T. Y. P., Mutasa-Göttgens, E. S., Dohm, J. C., Himmelbauer, H., Weisshaar, B., Kraus, J., Gielen, J. J. L., Lommel, M., Weyens, G., Wahl, B., Schechert, A., Nilsson, O., Jung, C., Kraft, T., and Müller, A. E. (2012). The role of a pseudo-response regulator gene in life cycle adaptation and domestication of beet. Current Biology, 22(12):1095-1101.

Putterill, J., Robson, F., Lee, K., Simon, R., and Coupland, G. (1995). The CONSTANS gene of Arabidopsis promotes flowering and encodes a protein showing similarities to zinc finger transcription factors. Cell, 80(6):847-857.

Putterill, J. and Varkonyi-Gasic, E. (2016). FT and florigen long-distance flowering control in plants. Current Opinion in Plant Biology, 33:77-82.

Putterill, J., Zhang, L., Yeoh, C. C., Balcerowicz, M., Jaudal, M., and Varkonyi-Gasic, E. (2013). FT 
genes and regulation of flowering in the legume Medicago truncatula. Functional Plant Biology, 40:1199-1207.

R Core Team (2018). R: A Language and Environment for Statistical Computing. R Foundation for Statistical Computing, Vienna, Austria.

Ridge, S., Deokar, A., Lee, R., Daba, K., Macknight, R. C., Weller, J. L., and Tar'an, B. (2017). The chickpea Early Flowering 1 (Efl1) locus is an ortholog of Arabidopsis ELF3. Plant Physiology, 175:802-815.

Ridge, S., Sussmilch, F. C., Hecht, V., Schoor, K. V., Lee, R., Aubert, G., Burstin, J., Macknight, R. C., and Weller, J. L. (2016). Identification of LATE BLOOMER2 as a CYCLING DOF FACTOR homolog reveals conserved and divergent features of the flowering response to photoperiod in pea. The Plant Cell, 28:2545-2559.

Roberts, E. H., Hadley, P., and Summerfield, R. J. (1985). Effects of temperature and photoperiod on flowering in chickpeas (Cicer arietinum L.). Annals of Botany, 55:881-892.

Shaw, L. M., Turner, A. S., Herry, L., Griffiths, S., and Laurie, D. A. (2013). Mutant alleles of Photoperiod1 in Wheat (Triticum aestivum L.) that confer a late flowering phenotype in long days. PLoS ONE, 8(11):e79459.

Simon, S., Rühl, M., de Montaigu, A., Wötzel, S., and Coupland, G. (2015). Evolution of CONSTANS regulation and function after gene duplication produced a photoperiodic flowering switch in the Brassicaceae. Molecular Biology and Evolution, 32(9):2284-2301.

Soneson, C., Love, M. I., and Robinson, M. D. (2015). Differential analyses for RNA-seq : transcript-level estimates improve gene-level inferences. F1000Research, 4:1521.

Song, Y. H., Kubota, A., Kwon, M. S., Covington, M. F., Lee, N., Taagen, E. R., Laboy Cintrón, D., Hwang, D. Y., Akiyama, R., Hodge, S. K., Huang, H., Nguyen, N. H., Nusinow, D. A., Millar, A. J., Shimizu, K. K., and Imaizumi, T. (2018). Molecular basis of flowering under natural long-day conditions in Arabidopsis. Nature Plants, 4(10):824-835.

Song, Y. H., Shim, J. S., Kinmonth-Schultz, H. A., and Imaizumi, T. (2015). Photoperiodic flowering: time measurement mechanisms in leaves. Annual Review of Plant Biology, 66:441-464.

Summerfield, R. J., Roberts, E. H., Erskine, W., and Ellis, R. H. (1985). Effects of temperature and photoperiod on flowering in lentils (Lens culinaris Medic .). Annals of Botany, 56:659-671.

Tadege, M., Wen, J., He, J., Tu, H., Kwak, Y., Eschstruth, A., Cayrel, A., Endre, G., Zhao, P. X., Chabaud, M., Ratet, P., and Mysore, K. S. (2008). Large-scale insertional mutagenesis using the Tnt1 retrotransposon in the model legume Medicago truncatula. The Plant Journal, 54(2):335-347.

Tang, H., Krishnakumar, V., Bidwell, S., Rosen, B., Chan, A., Zhou, S., Gentzbittel, L., Childs, K. L., Yandell, M., Gundlach, H., Mayer, K. F., Schwartz, D. C., and Town, C. D. (2014). An improved genome release (version Mt4.0) for the model legume Medicago truncatula. BMC Genomics, 15:312.

The Legume Phylogeny Working Group (2013). Legume phylogeny and classification in the 21st century: progress, prospects and lessons for other species-rich clades Legume phylogeny and classification in the 21st century: Progress, prospects and lessons for other species-rich clades. Taxon, 62:217-248.

Thomson, G. (2018). The transcriptomic response to a short day to long day shift in leaves of the reference legume medicago truncatula (code supplement). Figshare repository: https:// figshare.com/ articles/Thomson_et_al_2018_SDtoLDShiftAnalysis/6993641/4.

Turck, F., Fornara, F., and Coupland, G. (2008). Regulation and identity of florigen: FLOWERING LOCUS T moves center stage. Annual review of plant biology, 59:573-594.

Vance, C. P. (2001). Symbiotic nitrogen fixation and phosphorus acquisition. Plant nutrition in a world of declining renewable resources. Plant Physiology, 127:390-397.

Wahl, V., Ponnu, J., Schlereth, A., Arrivault, S., Langenecker, T., Franke, A., Feil, R., Lunn, J. E., Stitt, M., and Schmid, M. (2013). Regulation of flowering by trehalose-6-phosphate signaling in Arabidopsis thaliana. Science, 339:704-707.

Weller, J. L. and Ortega, R. (2015). Genetic control of flowering time in legumes. Frontiers in Plant Science, 6:207.

Wickham, H. (2017). Tidyverse: Easily Install and Load the 'Tidyverse'. R package version 1.2.1.

Wickland, D. P. and Hanzawa, Y. (2015). The FLOWERING LOCUS T/TERMINAL FLOWER 1 gene family: functional evolution and molecular mechanisms. Molecular Plant, 8(7):983-997.

Wigge, P. A., Kim, M. C., Jaeger, K. E., Busch, W., Schmid, M., Lohmann, J. U., and Weigel, D. (2005). Integration of spatial and temporal information during floral induction in Arabidopsis. Science, 
309(5737):1056-1059.

Wong, A. C. S., Hecht, V. F. G., Picard, K., Diwadkar, P., Laurie, R. E., Wen, J., Mysore, K., Macknight, R. C., and Weller, J. L. (2014). Isolation and functional analysis of CONSTANS-LIKE genes suggests that a central role for CONSTANS in flowering time control is not evolutionarily conserved in Medicago truncatula. Frontiers in Plant Science, 5:486.

Wu, F., Price, B. W., Haider, W., Seufferheld, G., Nelson, R., and Hanzawa, Y. (2014). Functional and evolutionary characterization of the CONSTANS gene family in short-day photoperiodic flowering in soybean. PLoS ONE, 9(1):e85754.

Xia, Z., Watanabe, S., Yamada, T., Tsubokura, Y., Nakashima, H., Zhai, H., Anai, T., Sato, S., Yamazaki, T., Lu, S., Wu, H., Tabata, S., and Harada, K. (2012). Positional cloning and characterization reveal the molecular basis for soybean maturity locus E1 that regulates photoperiodic flowering. PNAS, 109(32):E2155-E2164.

Yan, Y., Shen, L., Chen, Y., Bao, S., Thong, Z., and Yu, H. (2014). A MYB-domain protein EFM mediates flowering responses to environmental cues in Arabidopsis. Developmental Cell, 30:437-448.

Young, N. D., Debellé, F., Oldroyd, G. E. D., Geurts, R., Cannon, S. B., Udvardi, M. K., Benedito, V. A., Mayer, K. F. X., Gouzy, J., Schoof, H., Van de Peer, Y., Proost, S., Cook, D. R., Meyers, B. C., Spannagl, M., Cheung, F., De Mita, S., Krishnakumar, V., Gundlach, H., Zhou, S., Mudge, J., Bharti, A. K., Murray, J. D., Naoumkina, M. A., Rosen, B., Silverstein, K. A. T., Tang, H., Rombauts, S., Zhao, P. X., Zhou, P., Barbe, V., Bardou, P., Bechner, M., Bellec, A., Berger, A., Bergès, H., Bidwell, S., Bisseling, T., Choisne, N., Couloux, A., Denny, R., Deshpande, S., Dai, X., Doyle, J. J., Dudez, A.-M., Farmer, A. D., Fouteau, S., Franken, C., Gibelin, C., Gish, J., Goldstein, S., González, A. J., Green, P. J., Hallab, A., Hartog, M., Hua, A., Humphray, S. J., Jeong, D.-H., Jing, Y., Jöcker, A., Kenton, S. M., Kim, D.-J., Klee, K., Lai, H., Lang, C., Lin, S., Macmil, S. L., Magdelenat, G., Matthews, L., McCorrison, J., Monaghan, E. L., Mun, J.-H., Najar, F. Z., Nicholson, C., Noirot, C., O’Bleness, M., Paule, C. R., Poulain, J., Prion, F., Qin, B., Qu, C., Retzel, E. F., Riddle, C., Sallet, E., Samain, S., Samson, N., Sanders, I., Saurat, O., Scarpelli, C., Schiex, T., Segurens, B., Severin, A. J., Sherrier, D. J., Shi, R., Sims, S., Singer, S. R., Sinharoy, S., Sterck, L., Viollet, A., Wang, B.-B., Wang, K., Wang, M., Wang, X., Warfsmann, J., Weissenbach, J., White, D. D., White, J. D., Wiley, G. B., Wincker, P., Xing, Y., Yang, L., Yao, Z., Ying, F., Zhai, J., Zhou, L., Zuber, A., Dénarié, J., Dixon, R. A., May, G. D., Schwartz, D. C., Rogers, J., Quétier, F., Town, C. D., and Roe, B. A. (2011). The Medicago genome provides insight into the evolution of rhizobial symbioses. Nature, 480(7378):520-524. 
